\title{
1 Molecular and cellular insight into Escherichia coli SslE and its role during biofilm maturation
}

Paula M. Corsini ${ }^{1,2 \dagger}$, Sunjun Wang ${ }^{1 \dagger}$, Saima Rehman ${ }^{1 \dagger}$, Katherine Fenn ${ }^{2 \dagger}$, Amin Sagar $^{3}$, Slobodan 4 Sirovica $^{4}$, Leanne Cleaver ${ }^{1}$, Charlotte J. C. Edwards-Gayle ${ }^{5}$, Giulia Mastroianni, Ben Dorgan ${ }^{1}$, Lee M. Sewell ${ }^{1}$, Steven Lynham ${ }^{6}$, Dinu Iuga ${ }^{7}$, W. Trent Franks ${ }^{7}$, James Jarvis ${ }^{8}$, Guy H. Carpenter ${ }^{1}$, Michael.

${ }^{I}$ Centre for Host-Microbiome Interactions, Faculty of Dental, Oral \& Craniofacial Sciences, King's College London, London, $U K$

$9 \quad{ }^{2}$ School of Biological and Chemical Sciences, Queen Mary University of London, London, UK

$10 \quad{ }^{3}$ Centre de Biologie Structurale. INSERM, CNRS, Université de Montpellier, France

$11{ }^{4}$ Centre for Oral Bioengineering, Institute of Dentistry, Barts and The London School of Medicine and Dentistry, Queen Mary 12 University of London, London, $U K$

$13{ }^{5}$ Diamond Light Source Ltd., Diamond House, Harwell Science and Innovation Campus, Didcot, Oxfordshire, UK

$14{ }^{6}$ Proteomics Facility, Centre of Excellence for Mass Spectrometry, King's College London, London, UK

$15{ }^{7}$ Department of Physics, University of Warwick, Coventry, UK

$16{ }^{8}$ Randall Division of Cell and Molecular Biophysics and Centre for Biomolecular Spectroscopy, King's College London, London, $U K$

†equal contributions

*Correspondence to:

James Garnett, Centre for Host Microbiome Interactions, Dental institute, King's College London, London, UK. email: james.garnett@kcl.ac.uk 
Abstract

Escherichia coli is a Gram-negative bacterium that colonizes the human intestine and virulent strains can cause severe diarrhoeal and extraintestinal diseases. The protein SslE is secreted by a range of pathogenic and some commensal $E$. coli strains. It can degrade mucins in the intestine, promotes biofilm maturation and it is a major determinant of infection in virulent strains, although how it carries out these functions is not well understood. Here we examine SslE from the E. coli Waksman and $\mathrm{H10407}$ strains and reveal that it has a novel and dynamic structure. In response to acidification within mature biofilms we show how SslE forms a unique functional aggregate that interacts with cellulose and regulates the distribution of exopolysaccharides in macrocolony biofilms. Our data indicates that the spatial organization of SslE polymers and local pH are critical for biofilm maturation and SslE is a key factor that drives persistence of SslEsecreting bacteria during acidic stress.

Escherichia coli is a primary colonizer of the lower intestinal tract of humans and other warm-blooded animals. While many strains are considered beneficial to the host and help to maintain a healthy immune system, virulent strains are the cause of severe diarrhoeal diseases, including haemorrhagic colitis, and extraintestinal diseases such as neonatal meningitis, urinary tract infections, sepsis and pneumonia ${ }^{1}$. A wide range of pathogenic E. coli strains, and some commensals, use a Vibrio-like type II secretion system (T2SS) to translocate the protein SslE across their outer membrane and onto their extracellular surface $^{2,3,4,5,6,7}$. These include the Waksman (W), enterotoxigenic (ETEC), enterohemorrhagic (EHEC), enteropathogenic (EPEC), enteroaggregative (EAEC), enteroinvasive (EIEC) and neonatal meningitis $E$. coli (NMEC) strains. SslE is required for full virulence in a rabbit model of EPEC infection $^{4}$ and as a surface exposed antigen, SslE has shown great promise as a broadly protective vaccine candidate against a wide range of $E$. coli pathotypes ${ }^{3,6,8,9}$. 
60 SslE interacts with mucosal membranes in the host intestine where it can degrade mucins ${ }^{6,10,11,12}$, a 61 family of heavily $O$-linked glycosylated proteins and the primary constituents of mucus ${ }^{13}$. This provides 62 nutrients during bacterial growth but also enables these $E$. coli strains to penetrate the gut mucosa to 63 access host cells for efficient colonisation and targeting of toxins/effectors. Furthermore, SslE is also 64 important for mediating maturation of EPEC biofilms ${ }^{4}$; microbial aggregations encased within a selfproduced extracellular matrix comprised of exopolysaccharides, adhesive proteins and nucleic acids ${ }^{14}$. When E. coli is released into the environment through faeces or wastewater effluent it can survive for long periods within complex biofilm communities ${ }^{15}, 16$, and these are fundamental for both the environmental ecology of E. coli but also for successful colonisation of the intestinal tract ${ }^{17}$. However,

69 the specific molecular mechanisms that SslE uses to promote ecology and disease are not well understood.

SslE is a $\sim 165 \mathrm{kDa}$ lipoprotein composed of an $\mathrm{N}$-terminal periplasmic localization sequence and lipobox motif, an unstructured $\sim 5 \mathrm{kDa}$ region, a $\sim 110 \mathrm{kDa}$ region with no significant primary sequence homology to any other known protein, and a $~ 50 \mathrm{kDa}$ M60-like aminopeptidase domain at its Cterminus ${ }^{18}$ (Fig. 1a). M60-like domains are metalloproteases that contain a zinc binding HExxH motif and an additional conserved catalytic glutamate residue, which cleaves the peptide backbone of mucinlike substrates. These and other related enzymes have been identified in both prokaryotic and eukaryotic microbes that interact with host mucosal membranes ${ }^{18}$ and structures of proteoglycan complexes suggest that interactions with both the mucin peptide and $O$-linked carbohydrate sidechains are important for specificity ${ }^{19}$ 20. The peptidase activity of the SsIE M60 domain is effective for the degradation of major mucins of the intestine (e.g. MUC2, MUC3, MUC5AC) $)^{6,10,11,12}$, however, very

81 little is known as to how SslE interacts with these mucins or the function of the remaining SslE sequence. (NMR) spectroscopy and biochemical analyses we show that SslE from the E. coli W strain is formed 
86

87

that SslE undergoes conformational changes under acidic conditions, and this leads to the formation of higher order structures, which we propose is mediated by the NT2 and NT3 regions and is decorated with NT1 domains. We directly observe acidification within mature E. coli W biofilms and determine that SslE fibres can bind cellulose, a major exopolysaccharide of many E. coli biofilms. Furthermore, we provide evidence that SsIE regulates the localization of exopolysaccharides during maturation of both $E$. coli W and ETEC strain H10407 biofilms and this is likely a general mechanism in all SslEsecreting bacteria.

\section{Results}

SslE forms aggregates with amyloid-like properties. Functional amyloids are important proteinaceous structures in biofilms ${ }^{21}$ and analysis of the SslE primary sequence had previously highlighted several regions across the protein that may form amyloid-like structures ${ }^{22}$. Furthermore, environmental $\mathrm{pH}$ plays a major role initiating the formation of amyloid-like structures in Staphylococcus aureus, Enterococcus faecalis and Streptococcus mutans biofilms ${ }^{23,24,25}$, and we speculated that $\mathrm{pH}$ may also stimulate similar structural changes in SsIE. SslE from E. coli W, minus the $\mathrm{N}$-terminal lipidation motif and adjacent disordered region (rSslE; residues 67 to 1497; UniProt ID E0IW31) (Fig. 1a), was therefore produced in E. coli K-12 and purified by nickel-affinity and size exclusion chromatography. We then incubated $\mathrm{rSslE}$ across the $\mathrm{pH}$ range 4.0 to 7.0 and observed a ring of protein deposited on the walls of the tube at $\mathrm{pH} \leq 4.4$, which resisted solubilization up to $\mathrm{pH} 6.2$ (Fig. 1b). Mass spectrometry analysis of the ring identified peptides that spanned the complete sequence of rSslE and indicated that the aggregate contained intact protein, rather than a degradation product (Supplementary Fig. 1). We next used the amyloid diagnostic dye Thioflavin-T (ThT) to assess the formation of rSslE aggregates in solution over time. We observed large increases in ThT fluorescence emission again across the $\mathrm{pH}$ range 4.0 to 4.4 , but no change was detected when rSslE was incubated above pH 4.4 (Fig. 1c). Likewise, when rSslE was boiled and treated with sodium dodecyl sulphate 
111 (SDS) and then analysed by immunoblotting, stable high molecular weight species were observed over

112 the same pH range (Fig. 1d).

113 A main feature of amyloids is the presence of a cross- $\beta$-sheet structure ${ }^{26}$ and so we first analysed the

114 secondary structure of soluble rSslE using far-UV circular dichroism (CD) spectroscopy. This

115 suggested that at $\mathrm{pH} 7.4 \mathrm{rSslE}$ is composed of approximately $30 \% \alpha$-helix, $12 \% \beta$-sheet and $45 \%$ coil,

116 while under acidic conditions and prior to aggregation, there is an $\sim 8 \%$ loss of helical secondary

117 structure with a $~ 5 \%$ increase in $\beta$-sheet structure (Fig. 1e; Supplementary Table 1). We next used

118 attenuated total reflectance (ATR) Fourier transform mid-infrared (FT-MIR) spectroscopy to probe the

119 secondary structure composition of soluble rSslE at pH 7.4 and rSslE fibres at pH 4.4. Examination of

120 the amide I absorption band showed peaks at approximately $1656 \mathrm{~cm}^{-1}$ and $1624 \mathrm{~cm}^{-1}$, respectively

121 (Supplementary Fig. 2). The shift in the amide I absorption band to a lower frequency indicated that a

122 larger and more rigid structure had assembled under acid conditions. Furthermore, the presence of both

123 a high-intensity absorption band at $\sim 1624 \mathrm{~cm}^{-1}$ and a low-intensity band at $\sim 1695 \mathrm{~cm}^{-1}$ in the $\mathrm{pH} 4.4$

124 sample was indicative of an increase in anti-parallel $\beta$-sheet structure in rSslE aggregates ${ }^{27}$ and is

125 consistent with previous FT-MIR based characterisations of other anti-parallel $\beta$-sheet amyloid fibres ${ }^{28}$

126 (Fig. 1f).

127 Biofilm maturation supports polymerisation of SslE. Congo red is a dye which binds extracellular

128 polymeric substances (EPS), including amyloids, and we used this to assess the role of SslE during the

129 formation of macrocolony biofilms. We created an isogenic $\Delta s s l E$ knockout mutant in E. coli W and

130 ETEC strain H10407 and compared these to their parental wild-type strains. When these mutants were

131 cultured on Congo red agar plates for 24 hrs, we observed no obvious differences, however, after 96

132 hrs the mutants exhibited an altered morphology with the dye retained within the centre of the colony

133 (Fig. 2a). Complementation of the $\Delta s s l E$ mutant in $E$. coli W with a plasmid containing intact $s s l E$

134 ( $\triangle$ sslE::sslE) was able to restore bacterial secretion of SslE and recover macrocolony morphology

135 similar to that of the parental wild-type (Fig. 2a; Supplementary Fig. 3). We also generated a plasmid

136 expressing SslE with a truncated C-terminus ( $\triangle$ sslE::sslEAM60; residues 1 to 1000) to examine the role 
137 of the M60-domain during colony formation, and this again restored SslE secretion and wild-type

138 colony morphology (Fig. 2a; Supplementary Fig. 3). This led us to speculate that SslE experiences

139 extracellular $\mathrm{pH}$ values $<5.0$ during the development of biofilms and this results in SslE being deposited

140 as a functional aggregate.

141 To test this, we first grew E. coli $\mathrm{W}$ biofilms using a microfluidic system under continuous flow and 142 assessed their overall morphology using confocal laser scanning microscopy (CLSM). While biofilms 143 grown for $48 \mathrm{hrs}$ produced a relatively homogenous lawn of bacterial growth, after 96 hrs there were 144 clear signs of maturation, with a significant increase in biofilm mass, structural heterogeneity and the 145 presence of water channels (Fig. 2 b,c). We then examined the E. coli W $\Delta s s l E$ mutant under these 146 conditions, but in line with previous reports in EPEC strain E2348/69 ${ }^{4}$, it was unable to develop 147 structures beyond microcolonies (Fig. 2c,d). Furthermore, complementation of the mutant with either 148 intact $s s l E$ or $s s l E \Delta M 60$ was again able to restore wild-type biofilm morphology. Together with our 149 previous observation, this indicated that the $\mathrm{C}$-terminal M60-domain is not required for translocation of SslE through its T2SS and it is dispensable for biofilm development, at least under these conditions.

151 We then examined $\mathrm{pH}$ distribution across E. coli $\mathrm{W}$ biofilms using CLSM coupled with the cellimpermeant fluorescent ratiometric probe seminaphthorhodafluor-4F 5-(and-6) carboxylic acid (CSNARF-4). After growing E. coli $\mathrm{W}$ for $24 \mathrm{hrs}$ under continuous flow, we observed $\mathrm{pH}$ values between 6.0 and 6.3 across biofilms (Supplementary Fig. 4), however, after 96 hrs, lower pH values were also recorded (Fig. 2e). Although across the majority of biofilms examined, we detected $\mathrm{pH}$ values between approximately 5.0 and 6.0, clearly defined microenvironments were also observed with $\mathrm{pH}$ values that ranged from 4.2 and 4.8 .

We observed that when E. coli $\mathrm{W}$ was grown overnight in liquid culture at $\mathrm{pH} 5.0$, the wild-type strain and both $\Delta s s l E:: s s l E$ and $\triangle s s l E:: s s l E \Delta M 60$ complementation strains formed a clear ring on the wall of the tube that retained congo red dye (Fig. 3a). This was absent in the $\Delta s s l E$ mutant or when grown at

$161 \mathrm{pH} 7.0$ and mass spectrometry analysis of the wild-type E. coli W ring again identified SslE peptides

162 from across its sequence (Supplementary Fig. 1). Examination of wild-type E. coli $\mathrm{W}$ at pH 5.0 by 
163 immunoelectron microscopy revealed gold-labelled anti-rSslE antibodies associated with the bacterial

164 outer membrane, but also associated with fibrous material in the extracellular milieu and emanating

165 away from the bacterial surface; which was not observed in the $\Delta s s l E$ mutant (Fig. 3b,c,d). Inspection

166 of the E. coli $\mathrm{W} \Delta s s l E:: s s l E$ complemented strain under the same conditions produced less clustering

167 of anti-rSslE antibodies, however, a wild-type phenotype was observed when cells were incubated at

168 pH 4.0 prior to fixation and staining (Fig. 3e,f). This indicated that SslE can form aggregates upon

169 secretion from E. coli and so we then used immunofluorescence to assess how SslE is distributed within

170 established wild-type E. coli W and H10407 biofilms (Fig. 4). Here we observed a heterogenous

171 distribution and clustering of SslE across biofilms formed by both strains, with SslE co-localised with

172 bacteria, likely through association with the bacterial surface, but also clearly incorporated within the

173 extracellular matrix.

174 SslE aggregates bind cellulose. We speculated that during the maturation of biofilms, SslE aggregates interact with other components of the biofilm matrix and may contribute to the structural integrity of these communities. We therefore re-examined the morphology of wild-type and $\Delta s s l E$ mutant $E$. coli

177 W and H10407 macrocolonies using the carbohydrate stain calcofluor white, which binds $\beta 1-3$ and $\beta 1$ -

1784 polysaccharides including cellulose (Fig. 5a). As with Congo red staining, calcofluor white extended

179 to the edge of the colony in wild-type strains but was retained within the centre in the $\Delta s s l E$ mutants.

180 We then produced $\mathrm{rSslE}$ aggregates at $\mathrm{pH} 4.0$ and isolated them in citrate-phosphate buffer at $\mathrm{pH}$ 6.0,

181 a condition in which fibres would resist solubilization (Fig. 1b). As controls, we used monomeric rSslE,

182 and the C-terminal domain (CTD) of the Porphyromonas gingivalis gingipain protein RgpB, both of

183 which were not expected to bind cellulose or form polymers at this pH (Supplementary Fig. 5). We

184 then assayed binding of rSslE fibres to immobilised cellulose discs and observed a significant

185 interaction, but as expected this was not detected with monomeric rSslE or RgpB-CTD (Fig. 5b).

186 Overall structural features of SslE. We next initiated structural studies of SslE. Small angle X-ray 187 scattering (SAXS) coupled with size exclusion chromatography (SEC-SAXS) was first used to get 188 shape information for $\mathrm{rSslE}$ at $\mathrm{pH}$ 7.4. Guinier analysis provided a radius of gyration $\left(R_{\mathrm{g}}\right)$ of $4.03 \mathrm{~nm}$ 
189 and examination of the distance distribution function $(P(r))$ suggested a maximum particle dimension

$190 \quad\left(D_{\max }\right)$ of $14.1 \mathrm{~nm}$ and $R_{\mathrm{g}}$ of $4.07 \mathrm{~nm}$ (Supplementary Fig. 6; Supplementary Table 2). Calculation

191 of the particle molecular mass $(151 \mathrm{kDa})$ was consistent with a monomer in solution (theoretical mass

$192160 \mathrm{kDa}$ ). Ab initio dummy residue reconstruction generated 20 reproducible models with an average

193 normalized spatial discrepancy (NSD) score between reconstructions of 0.56 and a $\chi^{2}$ fit between

194 calculated and experimental solution scattering of 1.2 (Fig. 6a,b). The averaged low-resolution bead

195 model suggested that rSslE has a slightly elongated structure, however, examination of the Kratky plot

196 indicated that rSslE is a dynamic particle (Supplementary Fig. 6) and no obvious domain features

197 could be assigned.

198 Negative-stain TEM data was next collected for rSslE at $\mathrm{pH} 7.4$ and we performed single particle 199 analysis to generate an initial low-resolution structure, with overall dimensions of approximately $2008 \times 8 \times 11.5 \mathrm{~nm}($ Fig. 6c; Supplementary Fig. 7). Here, three well defined regions could be identified: 201 two adjacent globular domains (regions 1 and 2) with approximate diameters of $4 \mathrm{~nm}$ and $4.5 \mathrm{~nm}$, respectively, and a torus shaped structure (region 3) with a $\sim 15 \AA ̊$ wide central channel and approximate

203 overall dimensions of $4.5 \times 6.5 \times 9 \mathrm{~nm}$ (Fig. 6d). Superposition of the SAXS bead model with the TEM

204 envelope supported SslE being dynamic in solution, with an apparent reduction of bead volume in all

205 three regions of the SAXS model. Docking analysis of a homology model of the M60 domain, based

206 on the IMPa M60 metalloprotease from Pseudomonas aeruginosa (PDB ID code $5 \mathrm{kdv}$ ) ${ }^{19}$, indicated that

207 the C-terminus of SslE is located at the base of region 3 (Fig. 6e). This suggested that the remaining

208 density in region 3 is composed of the SslE sequence directly N-terminal to the M60 domain and these

209 two regions pack against one another to form a stable structure around an inter-domain channel.

210 Furthermore, from this model the M60 HExxH active site motif faces into the channel and this could

211 be an important feature for its mucolytic activity. The location of the M60 domain in the TEM map was

212 also supported by previous reports where it was not possible to purify a folded recombinant SslE M60

213 fragment due to sample instability ${ }^{22}$. 
214 Secondary structure analysis ${ }^{29}$ of the SslE sequence indicated two potential inter-domain boundaries

215 within the $\mathrm{N}$-terminal region and we predicted that these represented regions 1 and 2 located at the

216 extreme N-terminus (NT) of SslE. We therefore renamed these as the NT1 (residues 67-211) and NT2

217 (residues 230-425) domains, respectively, and renamed the remaining sequence as NT3 (residues 426-

218 1000) (Fig. 6f). The NT1 and NT2 domains, along with the NT1-NT2 region (residues 67-425) and

219 NT3-M60 core (region 3; residues 426-1497) were then produced in E. coli K-12 and purified by nickel-

220 affinity and size exclusion chromatography. Examination of these constructs using analytical gel

221 filtration and nuclear magnetic resonance (NMR) spectroscopy showed that they were well folded

222 (Supplementary Fig. 8), and supported NT1, NT2 and NT3-M60 being well-defined structural

223 boundaries within SslE. When we examined intact rSslE by NMR, a ${ }^{1} \mathrm{H}^{15} \mathrm{~N}$ transverse relaxation

optimized spectroscopy (TROSY) HSQC spectrum yielded only $~ 250$ strong resonances with the remaining 1200 peaks being either very weak or completely absent (Fig. 6g). Furthermore, NMR relaxation measurements of these intense peaks provided an estimated correlation time consistent with an $\sim 80 \mathrm{kDa}$ domain $\left(\tau_{\mathrm{c}} \sim 35 \mathrm{~ns}\right.$ at $\left.37^{\circ} \mathrm{C}\right)$. Comparison of $\mathrm{rSslE}$ with ${ }^{1} \mathrm{H}^{15} \mathrm{~N}-\mathrm{HSQC}$ spectra from SslE subdomains also clearly showed that the rSslE NMR spectrum is dominated by the NT1 domain but with minor contributions from the NT2 domain (Fig. 6g; Supplementary Fig. 9). This further supported this region having significant independent motion with respect to the NT3-M60 core and we considered whether the NT1-NT2 region alone could be responsible for mediating SslE aggregation. We therefore repeated our ring assay with SsIE NT1-NT2 and the NT3-M60 constructs incubated across the $\mathrm{pH}$ range 4.0 to 7.0 . This resulted in significantly less protein being deposited on the walls of the tubes and for NT3-M60 this was over a wider pH range (Supplementary Fig. 10). This implied that both the NT1-NT2 and the NT3 regions are necessary for correct polymerisation of intact SslE.

236 Kinetics of SslE aggregation. We took advantage of SEC-SAXS to probe the global shape of rSslE at $237 \mathrm{pH} 4.4$ prior to fibre formation and both Guinier analysis $\left(R_{g} 3.92 \mathrm{~nm}\right)$ and examination of the distance 238 distribution function $\left(D_{\max } 13.7 \mathrm{~nm}\right)$ produced very similar values to $\mathrm{rSslE}$ at $\mathrm{pH} 7.4$ (Supplementary

239 Fig. 11; Supplementary Table 2). However, the presence of better-defined features in the SAXS profile at acidic $\mathrm{pH}$ and the differences at large q-values in the normalized Kratky plots were indicative 
241 of rSslE becoming more rigid under acidic conditions (Fig. 7a; Supplementary Fig. 12). $A b$

242 initio dummy residue reconstruction produced 20 models with an average NSD score of 0.45 and a $\chi^{2}$ fit

243 between calculated and experimental solution scattering of 1.0 (Fig. 7b; Supplementary Fig. 13).

244 Comparison of the averaged bead models indicated an increase in bead volume within the NT1 and

245 NT2 domains and adjacent NT3 region at pH 4.4, while little change was observed in the NT3-M60

246 core, which supported an increase in rigidity under acidic conditions that could trigger aggregation.

247 We then exploited the additive nature of SAXS along with its ability to examine structures over a large 248 size range to study the kinetics of fibre formation by rSslE. We acquired SAXS data at $\mathrm{pH} 4.4$ during 249 the course of aggregation for $11 \mathrm{hrs}$ and the resulting 23 profiles displayed a systematic upwards 250 intensity increase, indicating the presence of large aggregates in solution (Fig. 7c). The appearance of 251 these large species occurred relatively quickly as the upwards intensity increase was already observed 252 after $1 \mathrm{hr}$ and the increase of aggregated species was concomitant to the loss of the SAXS features linked to the monomeric species. Principal Component Analysis (PCA) indicated that the data set could be described as a two-component system (Supplementary Fig. 14) and we decomposed the timedependent data set along with the SAXS profile of the monomer, obtained using SEC-SAXS, with

256 COSMiCS. COSMiCS uses a chemometric approach to decompose SAXS data sets in a model-free manner to derive the pure SAXS curves of the co-existing species and their relative populations, which we used here to report on the fibrillation kinetics ${ }^{30}$. The COSMiCS decomposition, assuming the coexistence of two components, was able to adequately fit all the SAXS profiles with an average $\left\langle\chi^{2}\right\rangle$ of 1.5, although some deviations from the perfect fit were observed for the SAXS curves measured in the first $3 \mathrm{hrs}(\mathbf{F i g . ~ 7 c})$. This observation suggested the presence of a small population of a third species in

262 the initial steps of fibrillation but this putative third species could not be captured when considering a 263 three-species decomposition with COSMiCS.

264 The extracted profile of the smallest species, and the subsequently derived $R_{\mathrm{g}}(3.93 \mathrm{~nm})$ and $D_{\max }(13.3$ nm) values, were almost identical to the SEC-SAXS profile for $\mathrm{rSslE}$ at $\mathrm{pH} 4.4$ (Fig. 7d,e; 
267 fibrillation process. The extracted SAXS profile of the second component displayed the typical features 268 of a large particle and $P(r)$ analysis indicated it had an $R_{g}$ of $\sim 51.1 \mathrm{~nm}$ and $D_{\max }$ of $\sim 140 \mathrm{~nm}($ Fig. 7d,e;

269 Supplementary Table 2). Fractal fit analysis ${ }^{31,32}$ of the decomposed SAXS profiles also indicated that

270 rSslE aggregates into a structure that is fractal in nature (Supplementary Fig. 15). The radius of the

271 primary particle was determined to be $\sim 3.5 \mathrm{~nm}$ while the mass fractal and surface fractal dimensions were determined to be 2.9 and 3.0, respectively. Examination of the relative populations of the two species derived from the COSMiCS decomposition over time showed that after 30 min from initiating the measurements, the aggregated species already represented 50\% of the molar fraction (Fig. 7f). From this time point, this population continued to grow and reached a plateau after $\sim 10 \mathrm{hrs}$.

Structural model of SslE fibres. Negative-stain TEM was used to visualize purified rSslE fibres revealing two common morphologies (Fig. 8a). The first form appeared as short single, flexible fibres with a core structure measuring $\sim 4.5 \mathrm{~nm}$ wide by $\sim 110 \mathrm{~nm}$ in length and decorated with globular structures $\sim 4.5 \mathrm{~nm}$ in diameter (Fig. 8b,c). The second morphology again resembled a fibrous material but measured between 20 to $40 \mathrm{~nm}$ in width by 200 to $300 \mathrm{~nm}$ in length (Fig. $8 \mathbf{d}$ ) and appeared to be an aggregation of the smaller fibres. Further analysis of rSslE aggregates in solution by real-time multiangle light scattering (RT-MALS) supported these observations (Supplementary Fig. 16). We measured a smaller species with an average molecular mass of $5.4 \times 10^{3} \mathrm{kDa}\left( \pm 0.4 \times 10^{3}\right)$ and a larger more polydisperse species with an average molecular mass of $12 \times 10^{3} \mathrm{kDa}\left( \pm 0.8 \times 10^{3}\right)$. In addition, we measured an average $R_{\mathrm{g}}$ of $137.5 \mathrm{~nm}( \pm 1.7)$ and $139.8 \mathrm{~nm}( \pm 1.5)$ for these species, respectively, which is in line with $R_{\mathrm{g}}$ measurements using COSMiCS. Examination of rSslE fibres using solid state NMR (ssNMR) showed individual narrow signals (Fig. 8e,f), indicative of an ordered structure, and we could tentatively assign approximately 40 ordered residues to specific residue types. Although we could not assign these resonances to a specific sequence position in SslE, the pattern suggested that these residues were localised to more than one site in SslE.

291 As the SAXS fibrillation data at the $10 \mathrm{hr}$ time point had shown depletion of monomeric rSslE but also had reduced fractal properties compared with later time points (Fig. 7f), we discarded the low q region 
and used this data to carry out analysis of the fibre asymmetric unit. Geometrical shape analysis

294 suggested that $\mathrm{rSslE}$ fibres form a cylinder with radius $3.2 \mathrm{~nm}$ and height $16.5 \mathrm{~nm}$, and cross-sectional

295 analysis indicated a repetitive unit with a cross-sectional $R_{g}\left(R_{c}\right)$ of $4.78 \mathrm{~nm}$. The cross-sectional $P(r)_{C}$

296 gave a cross-sectional $D_{\max }$ of $14.0 \mathrm{~nm}$. Ab initio dummy residue reconstruction produced a final model

297 with a $\chi^{2}$ fit between calculated and experimental solution scattering of 1.6 (Fig. 8g; Supplementary

298 Fig. 17). The width of the repeating unit varied between $\sim 3.5$ and $\sim 5.5 \mathrm{~nm}$, which is consistent with our measurements of single fibres by TEM. As we could not identify peripheral globular domains in the

300 fibre bead model, this supported SslE fibres being formed from a central core structure, with folded and

301 dynamic domains presented on the surface (Fig. 8h). Comparison of the fibre reconstruction with bead

302 models derived from the rSslE TEM envelope also suggested that a single molecule of SslE would

303 satisfy the bead volume but only once the NT1 domain had been removed.

\section{Discussion}

306 In this study we have revealed that SslE is composed of four defined regions: two globular domains at the N-terminus (NT1 and NT2) and a central torus shaped core, where a NT3 domain packs against an

308 M60-like aminopeptidase. The N-terminal region (NT1, NT2 and NT3) is composed of a unique

309 primary sequence that has only been identified in SsIE and its homologs ${ }^{33}$, while the C-terminal M60-

310 like domain is found in a wide range of proteins secreted by both prokaryotic and eukaryotic

311 microorganisms that interact with mucosal membranes ${ }^{18,34}$. The atomic structures for three M60-like

312 domains in complex with $O$-glycopeptides have been described: BT4244 from Bacteroides 313 thetaiotaomicron (PDB ID code: 5kd2), IMPa from P. aeruginosa (PDB ID code: 5kdw) and ZmpB

314 from Clostridium perfringens (PDB ID code: $5 \mathrm{kdn})^{19}$. While the active site structures in these bacterial

315 enzymes are highly conserved, there are variations within the remaining M60 domain, which enables

316 them to recognize unique glycan sequences. Structural data is not available for intact BT4244 or ZmpB,

317 although they are thought to contain additional carbohydrate binding domains ${ }^{18}$ and it is likely that these

318 M60 augmentations have a role in the recognition of $O$-glycopeptide substrates. It also appears that the 
active site in the M60 domain of SslE faces into the NT3 domain and this implies that both the NT3

320 and M60 domains are responsible for the recognition of mucins in SslE. With significant flexibility of

321 the NT1 and NT2 region, these domains may also interact with mucin substrates and may promote

322 processivity.

323 Although SslE has a clear function in the processing of mucins, it is a unique member of the M60-like aminopeptidase family as it is also fundamental for the maturation of EPEC biofilms grown on plastics ${ }^{4}$. Furthermore, we have now shown that SslE is also required for the maturation of E. coli W and ETEC H10407 biofilms when grown statically on agar and under shear stress on glass substrates, where it is widely distributed throughout the extracellular matrix. We have demonstrated that this unusual function is due to $\mathrm{SslE}$ forming aggregates at $\mathrm{pH} \leq 4.4$; conditions which are also observed as microenvironments across mature wild-type $E$. coli W biofilms. It is well established that phenotypical heterogeneity

330 develops during the maturation of biofilms and direct measurement of extracellular pH within E. coli

331 PHL628 biofilms has also shown a heterogenous distributions of $\mathrm{pH}$, with values ranging between 5.0 and $7.0^{35}$. Therefore, it is very likely that SslE forms functional aggregates within transiently forming microenvironments across maturing and established biofilms, but then resists solubilisation after any increase in the local $\mathrm{pH}$.

335 Microbial amyloid structures are ubiquitous in biofilms and in gram negative bacteria most known 336 amyloid structures are synthesized by either the Curli or Fap secretion systems ${ }^{36,37,38,39}$. These form 337 through spontaneous aggregation of extended peptides and provide adhesion, cohesion and contribute 338 to the structural integrity of the biofilm matrix. Although our biochemical and biophysical analyses 339 suggests that under acidic condition rSslE displays some amyloid-like features, examination of 340 aggregates using negative-stain TEM has revealed short fibres associated with globular structures.

341 SAXS reconstructions of rSslE fibres has allowed additional analysis and suggests that the repetitive

342 fibre unit can accommodate a single NT2-NT3-M60 region, and we therefore propose a potential model

343 for the fibrillation of SslE into functional aggregates via end-to-end stacking (Fig. 8i). In this model,

344 the asymmetric unit of the fibre core is composed of the NT2, NT3 and M60 domains and the NT1 
345 domain is flexible and can move freely around the surface. Polymerisation of the fibre core is mediated

346 through $\sim 40$ residues within the NT2 and NT3 domains but not the M60 domain, but this would require

347 some rearrangement of the NT3/M60 interface.

348 FTIR analysis of rSslE aggregates provided an amide I maxima of $1624 \mathrm{~cm}^{-1}$, which is consistent with

349 an increase in anti-parallel $\beta$-sheet conformation and also lies within the range of amide I maxima for 350 other typical amyloid structures $\left(\text { e.g. } 1611-1630 \mathrm{~cm}^{-1}\right)^{40}$. However, larger and more rigid amyloid structures generally have peaks at lower frequencies, while native $\beta$-sheets show absorption bands with maxima between 1630-1643 $\mathrm{cm}^{-1}$. This suggests that the rSslE amide I band peak represents an amyloid structure which is relatively small and is not overly rigid, although it could alternatively originate from

354 fibre stacking/clumping effects. Binding of ThT to rSslE at acidic $\mathrm{pH}$ also supports a general increase in $\beta$-sheet content in fibres but could also indicate the formation of new cavities upon fibrillation ${ }^{41}$. It is therefore still unclear whether these changes reflect the formation of a continuous amyloid-like structure that runs the length of the fibre core or instead represent $\beta$-sheet augmentation between adjacent SslE molecules along the fibre, upon fibrillation of SslE.

We have shown that SslE influences the localization of exopolysaccharides during the development of macrocolony biofilms and SslE fibres can directly associate with cellulose, which is known to have a structural function in many E. coli biofilms and provides cohesion and elasticity ${ }^{42}$. Although the precise

362 role of SslE during biofilm development is still vague, it could have an architectural role acting as a

363 hub to promote binding between other EPS or it could regulate the correct deposition of EPS within the

364 biofilm. As monomeric SslE does not recognise cellulose, this suggests that new binding sites are 365 formed after its aggregation. The presence of a surface exposed domain also implies that this domain may be functional in binding cellulose or other yet to be identified ligands, or is functional under different conditions, for example through interacting with mucins during intestinal colonisation.

368 Likewise, the fibre core may still retain its mucolytic activity and could play a role in mucin restructuring during biofilm growth in the gut. 
370 Although both E. coli $\mathrm{W}$ and ETEC 10407 secrete SsIE, the W strain is a harmless commensal, while

371 ETEC is a major etiological agent in developing countries ${ }^{43}$. SsIE is also required for colonisation in a

372 rabbit model of EPEC infection ${ }^{4}$ and is actively transcribed during ETEC infection of mice ${ }^{6}$. Biofilm

373 growth and the production of enterotoxins are essential for the pathogenesis of ETEC-induced disease ${ }^{43}$

374 and secretion of SslE and the ETEC labile toxin is via the same type II secretion system ${ }^{4,44}$. This

375 suggests that SslE increases the virulence of $E$. coli pathotypes through its ability to promote biofilm

376 maturation and/or through its interactions with mucosal defences. Both enterotoxigenic and

377 enteropathogenic E. coli strains cause infection in the small intestine ${ }^{1}$ where the intraluminal $\mathrm{pH}$ ranges

378 between approximately 6.0 to $7.5^{45}$. However, E. coli possess an acid-tolerance response that supports

379 exponential growth at $\mathrm{pH}$ values as low as $\mathrm{pH} 4.2^{46}$ and due to the synthesis of organic acids by the

380 residing microbiota, during colonisation of the intestine E. coli will experience extracellular pH values

381 that range between 4.0 and $6.0^{47}$. Although this suggests that within the intestinal tract, conditions exist

382 where SslE could regulate biofilm development, it remains unclear whether this role is important for

383 infection or during extraintestinal survival. SslE represents a unique protein and further studies are now

384 required to understand its complete functions during ecology and disease.

\section{Methods}

\section{Bacterial strains and media}

388 All bacterial strains, plasmids and primers used in this study are listed in Supplementary

389 Table 3. Lysogeny broth (LB) medium contained (per litre) $10 \mathrm{~g}$ tryptone, $5 \mathrm{~g}$ yeast extract and $10 \mathrm{~g}$

$390 \mathrm{NaCl}$. Isotopically defined M9 minimal medium $\left(\mathrm{pH}\right.$ 7.4) contained (per litre) $6.0 \mathrm{~g} \mathrm{Na} 2 \mathrm{HPO}_{4} \cdot 7 \mathrm{H}_{2} \mathrm{O}$,

$3913 \mathrm{~g} \mathrm{KH}_{2} \mathrm{PO}_{4}, 0.5 \mathrm{~g} \mathrm{NaCl}, 0.12 \mathrm{~g} \mathrm{MgSO}_{4} \cdot 7 \mathrm{H}_{2} \mathrm{O}, 0.022 \mathrm{~g} \mathrm{CaCl}_{2}, 0.04 \mathrm{~g}$ thiamine, $8.3 \mathrm{mg} \mathrm{FeCl} \cdot 6 \mathrm{H}_{2} \mathrm{O}$,

$3920.5 \mathrm{mg} \mathrm{ZnCl}_{2}, 0.1 \mathrm{mg} \mathrm{CuCl}$, $0.1 \mathrm{mg} \mathrm{CoCl} \cdot 6 \mathrm{H}_{2} \mathrm{O}, 0.1 \mathrm{mg} \mathrm{H}_{3} \mathrm{BO}_{3}$ and $13.5 \mathrm{mg} \mathrm{MnCl}_{2} \cdot 6 \mathrm{H}_{2} \mathrm{O}$,

393 supplemented with $2 \mathrm{~g}\left[\mathrm{U}_{-}{ }^{13} \mathrm{C}_{6}\right]$ glucose and/or $0.7 \mathrm{~g}{ }^{15} \mathrm{NH}_{4} \mathrm{Cl}$. M9 media was made up in deuterium 
394 oxide for the production of perdeuterated protein samples and $\mathrm{pH}$ was adjusted using $1 \mathrm{M} \mathrm{NaOH}$

395 solution. All NMR isotopes were from Sigma.

\section{Gene deletion}

397 A non-polar deletion of $s s l E$ was constructed in E. coli W and H10407 strains by allelic exchange 398 with FLP recombination target (FRT)-flanked $\mathrm{Kan}^{\mathrm{r}}$, using a modified protocol ${ }^{48}$. This fragment was 399 amplified from E. coli strain JW5925-1 ${ }^{49}$ using Platinum Taq DNA polymerase (Promega) and primers 400 PC3 and PC4 with specificity for the regions flanking sslE. These exchanges were facilitated by the $\lambda$ 401 Red recombinase system carried on plasmid pKD46 and knockouts were confirmed by PCR and 402 sequencing.

\section{Plasmid construction}

404 Complementation plasmids pCPC1 and pCPC2 were generated by amplifying whole length sslE, or 405 sslE minus the M60 domain, from E. coli $\mathrm{W}$ gDNA using primer pairs $\mathrm{PC} 7 / \mathrm{PC} 8$ or PC7/PC9, 406 respectively. These were digested with HindIII/NheI (NEB), ligated into HindIII/NheI-digested pBad407 cm18 vector. Synthetic genes gPC4, gPC5, gPC6 and gPC7 were synthesized by Synbio Technologies 408 and cloned into pET28b vector using NcoI and XhoI restriction sites to create plasmids pPC2, pPC3, 409 pPC4 and pPC5, respectively (Supplementary Table 4). Plasmid pBD1 was created by amplification 410 of the RgpB-CTD from P. gingivalis W50 gDNA using primers BD1 and BD2. This was then cloned 411 into pET46 Ek/LIC vector through ligation independent cloning (Novagen).

\section{Microfluidic biofilm growth}

413 Biofilms were grown in a BioFlux 200 device (Labtech, UK) using a protocol adapted from the

414 manufacturer. Single colonies of E. coli W and its derivatives were resuspended in $10 \mathrm{ml}$ of LB (with 415 additional $50 \mu \mathrm{g} / \mathrm{ml}$ kanamycin for $\Delta$ ssle mutant and $50 \mu \mathrm{g} / \mathrm{ml}$ kanamycin, $25 \mu \mathrm{g} / \mathrm{ml}$ chloramphenicol 416 Assle::pCPC1 (sslE) and $\triangle$ ssle::pCPC2 (sslEAM60)) and incubated at $37^{\circ} \mathrm{C}$ with shaking (200 rpm) for 41716 hrs. All subsequent media contained appropriate antibiotics where required. The cultures were then 
418 diluted (1:100) in $10 \mathrm{ml}$ of $\mathrm{LB}$ to $\mathrm{OD}_{600 \mathrm{~nm}}$ of 0.5 . A 24 well Bioflux plate $\left(0-20 \mathrm{dyn} / \mathrm{cm}^{2}\right)$ was pre-

419 warmed to $37^{\circ} \mathrm{C}$ on a heated stage and microfluidic channels were incubated with prewarmed LB. The 420 channels were then inoculated by injecting $20 \mu 1$ of bacterial suspension into the output reservoir for 5 $421 \mathrm{~s}$ at $2 \mathrm{dyne} / \mathrm{cm}^{2}$. The microfluidic plate was incubated for $1 \mathrm{hr}$ at $37^{\circ} \mathrm{C}$ to allow bacteria to bind to the surface and then the flow reversed for $20 \mathrm{~s}$. Prewarmed LB was then added to the input reservoir, the flow of media was initiated at 2 dyne $/ \mathrm{cm}^{2}$ for $5 \mathrm{~min}$ and then decreased to 1 dyne $/ \mathrm{cm}^{2}$ for $18 \mathrm{hrs}$. The spent media was removed from the output reservoir, fresh prewarmed media was added to the input reservoir and the flow was lowered to $0.5 \mathrm{dyne} / \mathrm{cm}^{2}$ for up to $96 \mathrm{hrs}$.

\section{Biofilm pH measurement}

The ratiometric dye seminaphthorhodafluor-4F 5-(and-6) carboxylic acid (C-SNARF-4) was used to directly measure $\mathrm{pH}$ across biofilms within the Bioflux channel, using a modified method ${ }^{50}$. HEPES buffer was first adjusted between $\mathrm{pH} 3.2$ to 7.8 in 0.2 increments using $\mathrm{HCl}$, and $95 \mu$ of each respective solution was then mixed with $5 \mu \mathrm{l} 1 \mathrm{mM} \mathrm{C}$-SNARF-4, resulting in a final $50 \mu \mathrm{M}$ concentration of CSNARF-4. This solution was flushed into the Bioflux plate channel, pre-warmed at $37^{\circ} \mathrm{C}$ and the channels were imaged at x64 magnification using a DM-IRE2 confocal laser scanning microscope (Leica Microsystems Heidelberg GmbH, Germany) and the accompanying Leica Microsystems

434 Confocal Software (version 2.61 Build 1537). Simultaneous images were captured using an excitation wavelength of $488 \mathrm{~nm}$ and emission wavelengths of $580 \mathrm{~nm}$ and $640 \mathrm{~nm}$, in triplicate for each $\mathrm{pH}$ increment with a background image obtained after each acquisition. Acquired images were analysed using ImageJ Software (FIJI) ${ }^{51}$ by determining the average intensity of fluorescence and standard deviation of each channel, minus the background control. The intensity ratio for each $\mathrm{pH}$ was used to make a standard curve by plotting known $\mathrm{pH}$ against green/red ratio. 50 $\mu \mathrm{M}$ C-SNARF-4 was then

440 flushed into Bioflux plates containing E. coli W biofilm after either 24 or 96 hrs growth and five 441 separate images were acquired for each Bioflux channel to calculate the $\mathrm{pH}$ from different areas of the biofilm. The fluorescence intensity and standard deviation were recorded, and the ratiometric value was 
each box was used to define regions of interest (ROI) and an average measure of $\mathrm{pH}$ was calculated within each region. The average $\mathrm{pH}$ for ROIs within the biofilm fringes and centres is presented.

\section{Biofilm immunofluorescence}

447 Single colonies of wild-type E. coli W or H10407 were resuspended in $10 \mathrm{ml}$ of LB and incubated at $44837^{\circ} \mathrm{C}$ with shaking (200 rpm) for $16 \mathrm{hrs}$. Cultures were diluted (1:100) in prewarmed $\mathrm{LB}$ and $5 \mathrm{ml}$ was 449 injected onto borosilicate glass coupons held within FC310 flow cells (Biosurface Technology) and 450 then incubated for $1 \mathrm{hr}$. Flow of LB media was initiated at $0.4 \mathrm{ml} / \mathrm{min}$ and maintained for $96 \mathrm{hrs}$. 451 Coupons were removed and washed three times for 10 min each in PBS-Tween. Coupons were then blocked for $1 \mathrm{hr}$ in $2 \%(\mathrm{w} / \mathrm{v}) \mathrm{BSA}$, PBS-Tween at room temperature and incubated overnight at $4^{\circ} \mathrm{C}$ 453 with polyclonal anti-rSslE antibody (rabbit; Invitrogen), diluted 1:200 in 0.1\% (w/v) BSA, PBS-Tween.

454 After three 10 min washes in PBS-Tween, coupons were incubated in the dark for $1 \mathrm{hr}$ with $10 \mu \mathrm{M}$ 455 Syto9 (Invitrogen) and anti-rabbit Alexa Fluor 633 secondary antibody (goat; Invitrogen) diluted 1:500 in $0.1 \%(\mathrm{w} / \mathrm{v}) \mathrm{BSA}$, PBS-Tween. This was followed by three 10 min washes in the dark in PBS-Tween and then fixation for $15 \mathrm{~min}$ at room temperature in the dark with $3 \%(\mathrm{w} / \mathrm{v})$ paraformaldehyde, PBSTween. After incubation for $15 \mathrm{~min}$ in $10 \mathrm{mM}$ glycine, PBS-Tween, images were captured at 100x magnification using an excitation wavelength of $486 \mathrm{~nm}$ and $632 \mathrm{~nm}$, and emission wavelengths of 501

$460 \mathrm{~nm}$ and $647 \mathrm{~nm}$, respectively, with a DM-IRE2 confocal laser scanning microscope (Leica

461 Microsystems Heidelberg GmbH, Germany). Negative controls consisted of biofilms being incubated 462 with either no primary anti-rSslE antibody or no Alexa Fluor 633 secondary antibody.

464 Overnight cultures were incubated for $30 \mathrm{~min}$ in $20 \mathrm{mM}$ citrate phosphate buffer at either $\mathrm{pH} 4.0$ or 5.0 465 and then washed in the same buffer before fixing with the $3 \%(\mathrm{w} / \mathrm{v})$ paraformaldehyde for $1 \mathrm{hr}$. Cells were then loaded onto a Glow discharged carbon coated Ni grid (Agar Scientific) for 10 mins, washed with $50 \mathrm{mM}$ glycine and then blocked with 1\% (v/v) Natural Donkey Serum (Jackson Immunoresearch) for $30 \mathrm{~min}$. This was then incubated for $1 \mathrm{hr}$ with primary polyclonal rSslE antibody (rabbit; Invitrogen) 
470 Donkey Serum and then incubated for $1 \mathrm{hr}$ with gold-conjugated secondary antibody (donkey; Jackson

471 Immunoresearch) diluted 1:100 in blocking buffer. Washing was repeated with buffer only $\mathrm{ddH}_{2} \mathrm{O}$. The 472 sample was negatively stained with $2 \%$ (w/v) uranyl acetate for $30 \mathrm{~s}$, followed by two quick washes 473 with double-distilled water. The grid was air dried, and images were recorded on a JEM1230 (JEOLJapan) at $80 \mathrm{KV}$ with a Morada CCD camera, iTEM software (EMSIS).

Recombinant SslE (residues 67-1497; numbered based on mature sequence) was expressed and purified as described previously ${ }^{12}$. Likewise, SslE NT1 (residues 67-211), NT2 (residues 230-425), NT1-NT2 (residues 67-425), NT3-M60 (residues 426-1497) and RgpB-CTD were transformed into $E$. coli SHuffle cells (SslE; New England Biolabs) or BL21 (DE3) (RgpB-CTD; New England Biolabs), grown at $37^{\circ} \mathrm{C}$ in LB media (rSslE, RgpB-CTD: $100 \mu \mathrm{g} / \mathrm{ml}$ ampicillin; NT1, NT2, NT1-NT2, NT3M60: $100 \mu \mathrm{g} / \mathrm{ml}$ kanamycin) and expression induced with $0.5 \mathrm{mM}$ isopropyl-d-1-thiogalactopyranoside (IPTG) at an $\mathrm{OD}_{600 \mathrm{~nm}}$ of 0.6 , followed by growth overnight at $18^{\circ} \mathrm{C}$. Cells were resuspended in $20 \mathrm{mM}$ Tris- $\mathrm{HCl} \mathrm{pH} \mathrm{8,} 200 \mathrm{mM} \mathrm{NaCl}$, lysed by sonication and purified using nickel affinity chromatography followed by gel filtration with either a Superdex 75 (NT1, NT2, RgpB-CTD) or 200 (rSslE, NT1-NT2, NT3-M60) column (GE Healthcare).

\section{SEC-SAXS}

487 SAXS data were collected on beamline B21 ${ }^{52}$ at Diamond Light Source (DLS, Oxford, UK), United Kingdom at $25^{\circ} \mathrm{C} .60 \mu \mathrm{lof} \mathrm{rSslE}(10 \mathrm{mg} / \mathrm{ml})$ in $20 \mathrm{mM}$ Tris- $\mathrm{HCl} \mathrm{pH} 8,200 \mathrm{mM} \mathrm{NaCl}$ was applied to a KW403-4F column (Shodex) at $0.16 \mathrm{ml} / \mathrm{min}$, preequilibrated in $20 \mathrm{mM}$ citrate phosphate buffer, 200 $\mathrm{mM} \mathrm{NaCl}$ at either $\mathrm{pH} 4.4$ or 7.4 , and SAXS data were measured over a momentum transfer range of

$4910.003<q<0.44 \AA^{-1}$. Peak integration and buffer subtraction were performed in CHROMIXS ${ }^{53}$. The radius of gyration $\left(R_{g}\right)$ and scattering at zero angle $[I(0)]$ were calculated from the analysis of the Guinier 
$494 \mathrm{GNOM}^{53}$ yielding the maximum particle dimension $\left(D_{\max }\right)$. Ab initio low resolution shape restoration

495 was carried out by calculating 20 models in $\mathrm{DAMMIF}^{53}$, which were subsequently averaged using

496 DAMAVER ${ }^{53}$ and used as a staring model for refinement in DAMMIN ${ }^{53}$. An additional 20 models were

497 also calculated and averaged in DAMAVER ${ }^{53}$. CRYSOL ${ }^{53}$ was used to compare final rSsIE TEM

498 envelopes and models against the solution SAXS curve. Processing and refinement statistics can be

499 found in Supplementary Table 2.

500 TEM single particle analysis

$5014 \mu \mathrm{l}$ of $\mathrm{rSslE}(625 \mathrm{nM})$ in $50 \mathrm{mM}$ Tris- $\mathrm{HCl} \mathrm{pH} 8.0,150 \mathrm{mM} \mathrm{NaCl}$ was applied to previously glow-

502 discharged 300 mesh continuous carbon-coated copper grids (Agar Scientific Ltd) for 1 min and blotted

503 for excess liquid. $4 \mu \mathrm{l}$ of $2 \%$ (v/v) uranyl acetate was applied for staining for $1 \mathrm{~min}$. The excess liquid

504 was blotted and left to dry. Data was acquired using a JEOL JEM-2100 plus TEM operating at $200 \mathrm{kV}$

505 equipped with a OneView 16 Megapixel camera (Gatan). 50 micrographs were collected at a nominal

506 magnification of $50,000 \times$ with a pixel size of $2.1 \AA$ Apixel and a range of defocus from 1 to $3 \mu \mathrm{m}$. Data

507 were processed using Relion $3.1^{54}$. Defocus and astigmatism parameters were estimated using

508 CTFFIND4 ${ }^{55}$ in Relion 3.1. An initial dataset of 41,752 particles were autopicked using 2D class

509 averages generated using approx. 1500 manually picked particles as reference. After a few rounds of

510 2D classification ignoring CTF until the first peak, 10,988 particles were taken forward for an initial

511 model generation using 3D initial model in Relion 3.1 $1^{54}$. Following initial model generation, a few

512 rounds of 3D classification followed by 3D refinement was carried out. The final model was refined to

$51322 \AA$ using the gold standard FSC (0.143 criterion). PHYRE $2^{56}$ was used to analyse the SsIE sequence

514 (residues 67-1497; UniProt ID E0IW31) and generate a homology model for the C-terminal M60

515 domain (residues 962-1415) based on residues 421-894 of P. aueriginosa IMPa (PDB ID code 5kdv;

$516100 \%$ confidence, $21 \%$ identity $)^{19}$. This was then docked into the rSslE TEM envelope using UCSF

517 CHIMERA ${ }^{57}$.

\section{Solution NMR spectroscopy}


519 NMR measurements for $\mathrm{rSslE}$ were performed at $25^{\circ} \mathrm{C}$ on a $100 \mu \mathrm{M}{ }^{2} \mathrm{H}^{15} \mathrm{~N}$-labelled sample in $50 \mathrm{mM}$ $520 \mathrm{NaPO}_{4} \mathrm{pH} 7.4,100 \mathrm{mM} \mathrm{NaCl}, 10 \% \mathrm{D}_{2} \mathrm{O}$. NMR measurements for SslE NT1 (0.6 mM), SslE NT2 (1.3 $521 \mathrm{mM})$ and SslE NT1-NT2 $(0.8 \mathrm{mM})$ were performed at $25^{\circ} \mathrm{C}$ on ${ }^{15} \mathrm{~N}$-labelled samples in $50 \mathrm{mM} \mathrm{NaPO}_{4}$ $522 \mathrm{pH} 7.0,100 \mathrm{mM} \mathrm{NaCl}, 10 \% \mathrm{D}_{2} \mathrm{O}$. NMR measurements for RgpB-CTD $(0.3 \mathrm{mM})$ were performed at $37^{\circ} \mathrm{C}$ on a ${ }^{15} \mathrm{~N}$-labelled sample in $20 \mathrm{mM} \mathrm{NaPO}_{4} \mathrm{pH} 6.0,100 \mathrm{mM} \mathrm{NaCl}, 10 \% \mathrm{D}_{2} \mathrm{O}$. Transverse relaxation optimized spectroscopy (TROSY) based ${ }^{1} \mathrm{H}^{15} \mathrm{~N}-\mathrm{HSQC}$ experiment and $T_{1}$ and $T_{2}$ relaxation times for rSslE were recorded on a Bruker Avance III HD 950, equipped with a TXI cryoprobe. TROSY ${ }^{1} \mathrm{H}^{15} \mathrm{~N}-\mathrm{HSQC}$ experiment for SslE NT1-NT2 was recorded on a on a Bruker Avance III HD 800, equipped with a TCI cryoprobe. Standard ${ }^{1} \mathrm{H}^{15} \mathrm{~N}-\mathrm{HSQC}$ spectra of SslE NT1, SslE NT2 and RgpB-CTD were recorded on a Bruker Avance III HD 700, equipped with a TCI cryoprobe. Data were processed in NMRPIPE ${ }^{58}$ and analysed/visualized with ANALYSIS ${ }^{59}$ and NMRVIEW ${ }^{60}$.

\section{SAXS fibrillation analysis}

531 SAXS data were collected on beamline B21 ${ }^{52}$ at DLS (Oxford, UK) at $20^{\circ} \mathrm{C}$. Immediately prior to data collection, $10 \mathrm{mg} / \mathrm{ml} \mathrm{rSslE} \mathrm{in} 20 \mathrm{mM}$ Tris- $\mathrm{HCl} \mathrm{pH}$ 8, $200 \mathrm{mM} \mathrm{NaCl}$ was buffer exchanged into $20 \mathrm{mM}$

533 citrate-phosphate buffer, pH 4.4 using a PD10 column (GE Healthcare) and the flow through was used 534 as a buffer reference. Using a peristaltic pump and while constantly stirring, $5 \mathrm{ml}$ of $\mathrm{rSslE}(0.7 \mathrm{mg} / \mathrm{ml})$ 535 was circulated through the SAXS imaging cuvette and data were collected every 30 min over a 536 momentum transfer range of $0.004<q<0.4 \AA^{-1}$, with the initial scattering data captured at 30 min after 537 initiating fibre growth. Data collected over the course of $11 \mathrm{hrs,} \mathrm{consisting} \mathrm{of} 22$ profiles, was 538 decomposed using $\operatorname{COSMiCS}^{30}$, which utilizes MCR-ALS ${ }^{61}$ to perform model free decomposition of 539 the entire SAXS data set. The SEC-SAXS curve collected at $\mathrm{pH} 4.4$ was also included in the dataset as

540 the representative state of the protein at time $0 \mathrm{~s}$, i.e. before initiating fibrillation. The time $0 \mathrm{~s}$ curve 541 was selected as one of the initial estimates and the selectivity restraint was used to ascertain that the 542 curve had no contribution from the other species. In addition, non-negativity restraint was imposed for

543 both the SAXS profiles and concentrations using FNNLS algorithm ${ }^{62}$. Before COSMiCS analysis, 544 SAXS data were scaled according to the large angle data to enhance the decomposition capacity of the 
approach. Although COSMiCS was run assuming a two-component system as suggested by Principal

546 Component Analysis (PCA), a three-species run was also performed. The convergence criterion of <

$5470.01 \%$ change in lack of fit was used with 1,000 maximum allowed iterations. The analysis of the

548 resulting COSMiCS curves was performed with the ATSAS suit of programs ${ }^{53}$. Fractal analysis was

549 carried out using SASVIEW (http://www.sasview.org/).

\section{SAXS fibre modelling}

551 The programs ATSAS $^{53}$ and Scatter were used to obtain a cross-sectional radius of gyration $R_{c}$ from the

$55210 \mathrm{hr}$ post-fibre induction scattering profile and from this the cross-sectional $P(r)_{C}$ was calculated.

553 BODIES ${ }^{53}$ was then used to approximate the geometric shape of rSslE fibres, which suggested a

554 cylindrical shape with radius of $3.93 \mathrm{~nm}$ and a height of $16.5 \mathrm{~nm}\left(\chi^{2} 1.6\right)$. These dimensions were then

555 used in DAMMIN ${ }^{63}$ with data from the q range 0.03 to 0.20 to create ab initio shapes of the SslE fibril

556 repeat. 20 individual jobs were run, generating 20 independent models and were subsequently averaged

557 using DAMAVER ${ }^{53}$ and then refined 5 times in DAMMIN ${ }^{63}$. A bead model was produced from the

558 rSslE negative-stain TEM envelope using the program EM2DAM ${ }^{53}$, the NT1 bead region was removed

559 and then this was superposed onto the DAMMIN fibre bead model using SUPCOMB ${ }^{53}$. Processing and

560 refinement statistics can be found in Supplementary Table 2.

\section{TEM fibre analysis}

$562 \mathrm{rSslE}$ at $1 \mathrm{mg} / \mathrm{ml}$ was incubated in $100 \mathrm{mM}$ citrate phosphate $\mathrm{pH} 3.8$ overnight at room temperature

563 while shaking at $180 \mathrm{rpm}$. This was centrifuged at $15,000 \mathrm{~g}$ for $10 \mathrm{~min}$, the top $80 \%$ of buffer discarded

564 and then $4 \mu \mathrm{l}$ of the remaining sample was applied to a previously glow-discharged 300 mesh

565 continuous carbon-coated copper grids (Agar Scientific Ltd) and immediately blotted for excess liquid.

$5664 \mu \mathrm{l}$ of $2 \%(\mathrm{v} / \mathrm{v})$ uranyl acetate was applied for staining for 10 seconds. The excess liquid was blotted

567 and left to dry. Data was acquired using a JEOL JEM-1230 TEM operating at $80 \mathrm{kV}$ equipped with a

568 Morada 2k CCD camera system and its iTEM software package (Olympus Europa, UK). Micrographs

569 were collected at a nominal magnification of 80,000x with a pixel size of $5.96 \AA$ A $/$ pixel. 


\section{Solid state NMR spectroscopy}

$571 \mathrm{rSslE}$ at $20 \mathrm{mg} / \mathrm{ml}$ was buffer exchanged into $10 \mathrm{mM}$ citrate phosphate $\mathrm{pH} 4.0$ using a PD10 column

572 (Sigma) and then incubated overnight at room temperature while shaking at $180 \mathrm{rpm}$. This was

573 centrifuged at $15,000 \mathrm{~g}$ for $10 \mathrm{~min}$, the top $80 \%$ of buffer was discarded and then the remaining sample

574 was used for solid state NMR analysis. Experiments were performed using a Bruker Neo Console operating at $850 \mathrm{MHz}{ }^{1} \mathrm{H}$ frequency with a $3.2 \mathrm{~mm}$ E-Free probe in $\mathrm{HC}$ mode spinning at a rate $\mathrm{w}_{\mathrm{r}}=15$ kHz. A standard CP excitation ${ }^{13} \mathrm{C}-{ }^{13} \mathrm{C}$ DARR experiment was acquired. The direct dimension was acquired for $16.4 \mathrm{~ms}$ with $\sim 55 \mathrm{kHz}$ SPINAL-64 decoupling. 256 rows with 256 coadded transients and

578 a recovery delay of $2.5 \mathrm{~s}$ were acquired using Time-Proportional Phase Increment (TPPI) with a 22.2

$579 \mu$ s dwell (45 kHz sweep width, $2.84 \mathrm{~ms}$ total evolution) for a total of $45.5 \mathrm{hrs}$ total acquisition time.

580 The applied power was adjusted so that ${ }^{1} \mathrm{H}$ and ${ }^{13} \mathrm{C}$ hard pulses were both $4 \mu \mathrm{s}\left(\mathrm{w}_{1(\mathrm{H}, \mathrm{C})}=62.5 \mathrm{kHz}\right)$. The

581 initial carbon excitation was achieved with 1.5 ms of ramped Hartmann-Hahn CP, where the optimal 582 polarization transfer was found at $\mathrm{w}_{1 \mathrm{C}}=\sim 70 \mathrm{kHz}$ and $\mathrm{w}_{1 \mathrm{H}}=\sim 55 \mathrm{kHz}$ with an upwards linear ramp from $58370-100 \%$ on the ${ }^{1} \mathrm{H}$ channel. Homonuclear Carbon mixing was achieved with $50 \mathrm{~ms}$ of DARR mixing $584 \quad\left(\mathrm{w}_{1 \mathrm{H}}=\mathrm{w}_{\mathrm{r}}=15 \mathrm{kHz}\right)$.

\section{Acknowledgements}

587 PC was supported by the Academy of Medical Sciences and Wellcome Trust (SBF002/1150) and SR

588 was supported by the Medical Research Council (MR/R017662/1), awarded to JAG. SW and BD were

589 supported by China Scholarship Council and Biotechnology and Biological Sciences Research Council 590 studentships, respectively, awarded to JAG and MAC. LS was supported by the Leverhulme Trust 591 (RPG-2017-222), awarded to JAG. KF was supported by a PhD studentship from Queen Mary

592 University of London and VCD was supported by a start-up grant from Queen Mary University of 593 London. This work was also supported by the Labex EpiGenMed, an « Investissements d'avenir » 594 program (ANR-10-LABX-12-01) awarded to PB. The CBS is a member of France-BioImaging (FBI) 
and the French Infrastructure for Integrated Structural Biology (FRISBI), 2 national infrastructures supported by the French National Research Agency (ANR-10-INBS-04-01 and ANR-10-INBS-05, respectively). We thank the beamline scientists at B21 of the Diamond Light Source, United Kingdom.

598 This work was also supported by the Francis Crick Institute through provision of access to the MRC 599 Biomedical NMR centre. The Francis Crick Institute receives its core funding from Cancer Research 600 United Kingdom (FC001029), the United Kingdom Medical Research Council (FC001029), and the

601 Wellcome Trust (FC001029). We also thank the Centre for Biomolecular Spectroscopy at King's

602 College London for additional NMR access, funded by the Wellcome Trust and British Heart 603 Foundation (ref. 202767/Z/16/Z and IG/16/2/32273 respectively). The UK $850 \mathrm{MHz}$ solid-state NMR

604 Facility used in this research was funded by EPSRC and BBSRC (contract reference EP/T015063/1), 605 as well as the University of Warwick including via part funding through Birmingham Science City 606 Advanced Materials Projects 1 and 2 supported by Advantage West Midlands (AWM) and the European 607 Regional Development Fund (ERDF). Cellulose discs were gifted by Prof. Tom Ellis and Kenneth T. 608 Walker (Imperial College London).

611 Conceived and designed the experiments: PC, SW, SR, KF, SS, CE-G, AS, GM, BD, LMS, SL, DI, 612 PB, VCD, JAG. Performed the experiments: PC, SW, SR, KF, AS, SS, LC, CE-G, GM, BD, LMS, SL, 613 DI, JJ, GHC, VCD, JAG. Analyzed the data: PC, SW, SR, KF, AS, SS, CE-G, GM, BD, SL, DI, TF, 614 PB, VCD, JAG. Contributed reagents/materials/analysis tools: SS, SL, PB, VCD, JAG. Wrote the 615 paper: PC, SW, SR, KF, AS, SS, LC, CE-G, GM, BD, LMS, SL, DI, TF, JJ, GHC, MAC, PB, VCD, 616 JAG.

\section{References}


2. Gu S, Shevchik VE, Shaw R, Pickersgill RW, Garnett JA. The role of intrinsic disorder and dynamics in the assembly and function of the type II secretion system. Biochim Biophys Acta Proteins Proteom 1865, 1255-1266 (2017).

3. Moriel DG, et al. Identification of protective and broadly conserved vaccine antigens from the genome of extraintestinal pathogenic Escherichia coli. Proc Natl Acad Sci U S A 107, 9072-9077 (2010).

4. Baldi DL, et al. The type II secretion system and its ubiquitous lipoprotein substrate, SslE, are required for biofilm formation and virulence of enteropathogenic Escherichia coli. Infect Immun 80, 2042-2052 (2012).

5. Decanio MS, Landick R, Haft RJ. The non-pathogenic Escherichia coli strain W secretes SslE via the virulence-associated type II secretion system beta. BMC Microbiol 13, 130 (2013).

6. Nesta B, et al. SslE elicits functional antibodies that impair in vitro mucinase activity and in vivo colonization by both intestinal and extraintestinal Escherichia coli strains. PLoS Pathog 10, e1004124 (2014).

7. Yang J, Baldi DL, Tauschek M, Strugnell RA, Robins-Browne RM. Transcriptional regulation of the yghJ-pppA-yghG-gspCDEFGHIJKLM cluster, encoding the type II secretion pathway in enterotoxigenic Escherichia coli. J Bacteriol 189, 142-150 (2007).

8. Naili I, et al. Mixed mucosal-parenteral immunizations with the broadly conserved pathogenic Escherichia coli antigen SslE induce a robust mucosal and systemic immunity without affecting the murine intestinal microbiota. Vaccine 37, 314-324 (2019).

9. Luo Q, Qadri F, Kansal R, Rasko DA, Sheikh A, Fleckenstein JM. Conservation and immunogenicity of novel antigens in diverse isolates of enterotoxigenic Escherichia coli. PLoS Negl Trop Dis 9, e0003446 (2015).

10. Valeri M, et al. Pathogenic E. coli exploits SslE mucinase activity to translocate through the mucosal barrier and get access to host cells. PLoS One 10, e0117486 (2015).

11. Luo Q, et al. Enterotoxigenic Escherichia coli secretes a highly conserved mucin-degrading metalloprotease to effectively engage intestinal epithelial cells. Infect Immun 82, 509-521 (2014).

12. Rehman S, et al. Structure and functional analysis of the Legionella pneumophila chitinase ChiA reveals a novel mechanism of metal-dependent mucin degradation. PLoS Pathog 16, e1008342 (2020).

13. Corfield AP. Mucins: a biologically relevant glycan barrier in mucosal protection. Biochim Biophys Acta 1850, 236-252 (2015). 
14. Garnett JA, Matthews S. Interactions in bacterial biofilm development: a structural perspective. Curr Protein Pept Sci 13, 739-755 (2012).

15. Vogeleer P, Tremblay YD, Mafu AA, Jacques M, Harel J. Life on the outside: role of

671

672

673 Microbiol 5, 317 (2014).

16. Chitlapilly Dass S, et al. Impact of mixed biofilm formation with environmental

675 microorganisms on E. coli O157:H7 survival against sanitization. NPJ Sci Food 4, 16 (2020).

676

677

678

679

680

681

682

683

684

685

686

687

688

689

690

691

692

693

694

695

696

697

698

699

17. Rossi E, et al. "It's a gut feeling" - Escherichia coli biofilm formation in the gastrointestinal tract environment. Crit Rev Microbiol 44, 1-30 (2018).

18. Nakjang S, Ndeh DA, Wipat A, Bolam DN, Hirt RP. A novel extracellular metallopeptidase domain shared by animal host-associated mutualistic and pathogenic microbes. PLoS One 7, e30287 (2012).

19. Noach I, et al. Recognition of protein-linked glycans as a determinant of peptidase activity. Proc Natl Acad Sci U S A 114, E679-E688 (2017).

20. Trastoy B, Naegeli A, Anso I, Sjogren J, Guerin ME. Structural basis of mammalian mucin processing by the human gut O-glycopeptidase OgpA from Akkermansia muciniphila. Nat Commun 11, 4844 (2020).

21. Taglialegna A, Lasa I, Valle J. Amyloid Structures as Biofilm Matrix Scaffolds. J Bacteriol 198, 2579-2588 (2016).

22. Belousov MV, et al. M60-like metalloprotease domain of the Escherichia coli YghJ protein forms amyloid fibrils. PLoS One 13, e0191317 (2018).

23. Taglialegna A, et al. The biofilm-associated surface protein Esp of Enterococcus faecalis forms amyloid-like fibers. NPJ Biofilms Microbiomes 6, 15 (2020).

24. Taglialegna A, et al. Staphylococcal Bap Proteins Build Amyloid Scaffold Biofilm Matrices in Response to Environmental Signals. PLoS Pathog 12, e1005711 (2016).

25. Chen D, et al. Characteristics and influencing factors of amyloid fibers in S. mutans biofilm. AMB Express 9, 31 (2019).

26. Gallardo R, Ranson NA, Radford SE. Amyloid structures: much more than just a cross-beta fold. Curr Opin Struct Biol 60, 7-16 (2020). 
27. Moran SD, Zanni MT. How to Get Insight into Amyloid Structure and Formation from Infrared Spectroscopy. J Phys Chem Lett 5, 1984-1993 (2014).

711

28. Conway KA, Harper JD, Lansbury PT, Jr. Fibrils formed in vitro from alpha-synuclein and two mutant forms linked to Parkinson's disease are typical amyloid. Biochemistry 39, 25522563 (2000).

29. Buchan DWA, Jones DT. The PSIPRED Protein Analysis Workbench: 20 years on. Nucleic Acids Res 47, W402-W407 (2019).

718

30. Herranz-Trillo F, Groenning M, van Maarschalkerweerd A, Tauler R, Vestergaard B, Bernado P. Structural Analysis of Multi-component Amyloid Systems by Chemometric SAXS Data Decomposition. Structure 25, 5-15 (2017).

31. Schmidt P. Small-angle scattering studies of disordered, porous and fractal systems. Journal of Applied Crystallography 24, 414-435 (1991).

32. Hurd AJ, Schaefer DW, Martin JE. Surface and mass fractals in vapor-phase aggregates. Phys Rev A Gen Phys 35, 2361-2364 (1987).

33. Gish W, States DJ. Identification of protein coding regions by database similarity search. Nat Genet 3, 266-272 (1993).

37. Shanmugam N, Baker M, Ball SR, Steain M, Pham CLL, Sunde M. Microbial functional

34. Ta BTT, et al. Identification, recombinant protein production, and functional analysis of a M60-like metallopeptidase, secreted by the liver fluke Opisthorchis viverrini. Parasitol Int 75, 102050 (2020).

35. Hidalgo G, et al. Functional tomographic fluorescence imaging of $\mathrm{pH}$ microenvironments in microbial biofilms by use of silica nanoparticle sensors. Appl Environ Microbiol 75, 74267435 (2009).

36. Van Gerven N, Van der Verren SE, Reiter DM, Remaut H. The Role of Functional Amyloids in Bacterial Virulence. J Mol Biol 430, 3657-3684 (2018).

38. Dueholm MS, et al. Functional amyloid in Pseudomonas. Mol Microbiol 77, 1009-1020 (2010). 
39. Evans ML, Chapman MR. Curli biogenesis: order out of disorder. Biochim Biophys Acta 1843, 1551-1558 (2014).

40. Zandomeneghi G, Krebs MR, McCammon MG, Fandrich M. FTIR reveals structural differences between native beta-sheet proteins and amyloid fibrils. Protein Sci 13, 3314-3321 (2004).

41. Groenning M, Olsen L, van de Weert M, Flink JM, Frokjaer S, Jorgensen FS. Study on the binding of Thioflavin T to beta-sheet-rich and non-beta-sheet cavities. J Struct Biol 158, 358369 (2007).

42. Serra DO, Richter AM, Hengge R. Cellulose as an architectural element in spatially structured Escherichia coli biofilms. J Bacteriol 195, 5540-5554 (2013).

43. Qadri F, Svennerholm AM, Faruque AS, Sack RB. Enterotoxigenic Escherichia coli in developing countries: epidemiology, microbiology, clinical features, treatment, and prevention. Clin Microbiol Rev 18, 465-483 (2005).

44. Sjoling A, von Mentzer A, Svennerholm AM. Implications of enterotoxigenic Escherichia coli genomics for vaccine development. Expert Rev Vaccines 14, 551-560 (2015).

45. Fallingborg J. Intraluminal pH of the human gastrointestinal tract. Dan Med Bull 46, 183-196 (1999).

46. $\mathrm{Xu} \mathrm{Y,} \mathrm{et} \mathrm{al.} \mathrm{An} \mathrm{acid-tolerance} \mathrm{response} \mathrm{system} \mathrm{protecting} \mathrm{exponentially} \mathrm{growing} \mathrm{Escherichia}$ coli. Nat Commun 11, 1496 (2020).

47. Lin J, Smith MP, Chapin KC, Baik HS, Bennett GN, Foster JW. Mechanisms of acid resistance in enterohemorrhagic Escherichia coli. Appl Environ Microbiol 62, 3094-3100 (1996).

48. Datsenko KA, Wanner BL. One-step inactivation of chromosomal genes in Escherichia coli K-12 using PCR products. Proc Natl Acad Sci U S A 97, 6640-6645 (2000).

49. Baba T, et al. Construction of Escherichia coli K-12 in-frame, single-gene knockout mutants: the Keio collection. Mol Syst Biol 2, 20060008 (2006).

50. Schlafer S, Dige I. Ratiometric Imaging of Extracellular pH in Dental Biofilms. J Vis Exp, (2016).

51. Schindelin J, et al. Fiji: an open-source platform for biological-image analysis. Nat Methods 9, 676-682 (2012). 
52. Cowieson NP, et al. Beamline B21: high-throughput small-angle X-ray scattering at Diamond Light Source. J Synchrotron Radiat 27, 1438-1446 (2020).

53. Franke D, et al. ATSAS 2.8: a comprehensive data analysis suite for small-angle scattering from macromolecular solutions. J Appl Crystallogr 50, 1212-1225 (2017).

54. Zivanov J, et al. New tools for automated high-resolution cryo-EM structure determination in RELION-3. Elife 7, (2018).

55. Rohou A, Grigorieff N. CTFFIND4: Fast and accurate defocus estimation from electron micrographs. J Struct Biol 192, 216-221 (2015).

56. Kelley LA, Mezulis S, Yates CM, Wass MN, Sternberg MJ. The Phyre2 web portal for protein modeling, prediction and analysis. Nat Protoc 10, 845-858 (2015).

57. Pettersen EF, et al. UCSF Chimera--a visualization system for exploratory research and analysis. J Comput Chem 25, 1605-1612 (2004).

58. Delaglio F, Grzesiek S, Vuister GW, Zhu G, Pfeifer J, Bax A. NMRPipe: a multidimensional spectral processing system based on UNIX pipes. J Biomol NMR 6, 277-293 (1995).

59. Vranken WF, et al. The CCPN data model for NMR spectroscopy: development of a software pipeline. Proteins 59, 687-696 (2005).

60. Johnson BA, Blevins RA. NMR View: A computer program for the visualization and analysis of NMR data. J Biomol NMR 4, 603-614 (1994).

61. Jaumot J, Gargallo R, de Juan A, Tauler R. A graphical user-friendly interface for MCR-ALS: a new tool for multivariate curve resolution in MATLAB. Chemometrics and Intelligent Laboratory Systems 76, 101-110 (2005).

62. Bro R, De Jong S. A fast non-negativity-constrained least squares algorithm. Journal of Chemometrics 11, 393-401 (1997).

63. Volkov VV, Svergun DI. Uniqueness of ab initio shape determination in small-angle scattering. Journal of Applied Crystallography 36, 860-864 (2003). 


\section{Figures}

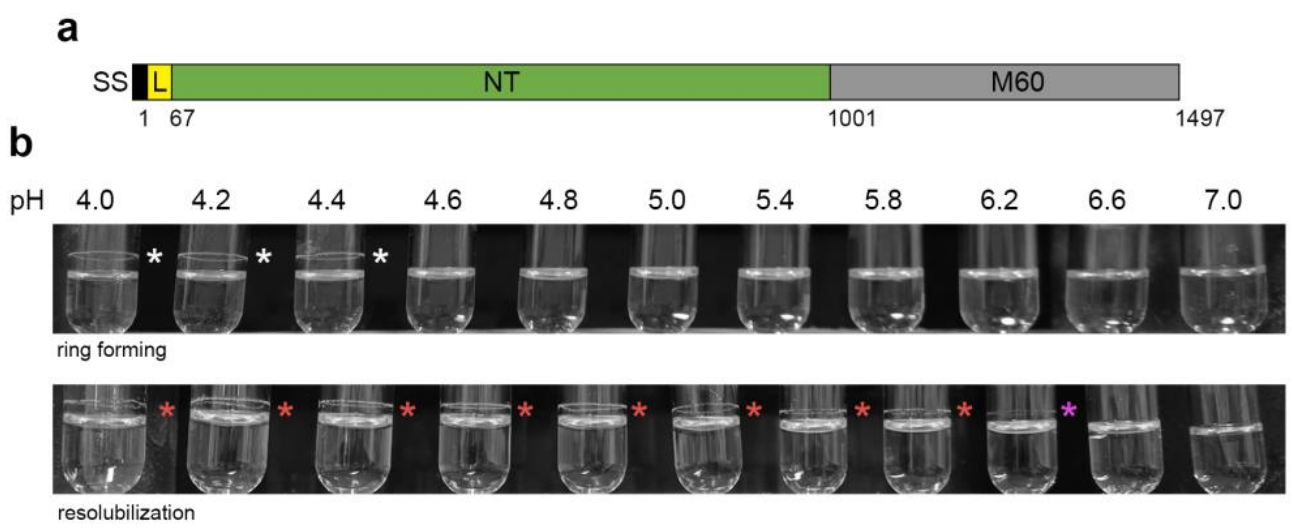

C
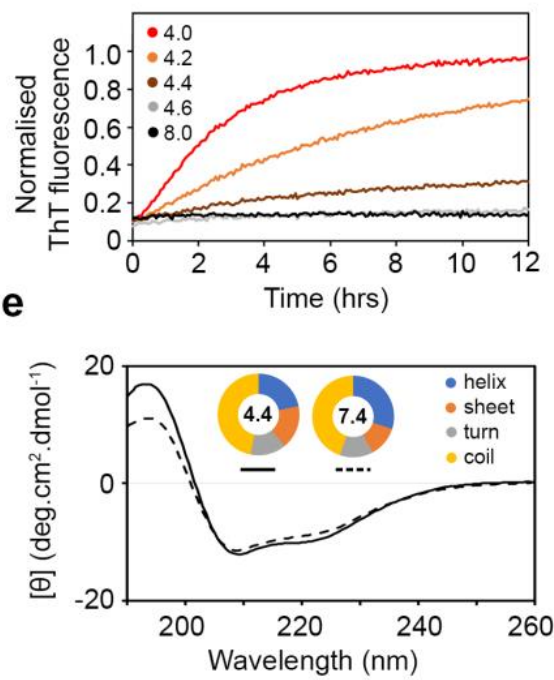

d
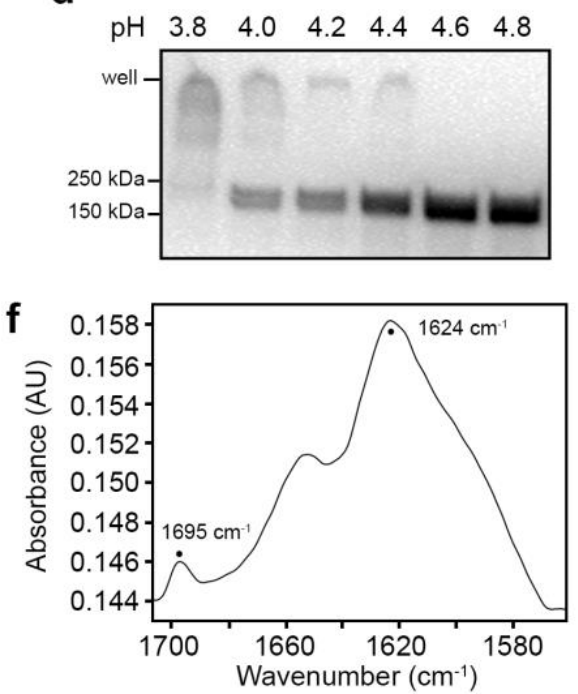

Fig. 1 | Characterization of SslE aggregates. a, Schematic of SslE from E. coli W with mature sequence numbers and structural features annotated. SS: periplasmic signal sequence; L: flexible linker; NT: unique N-terminal region; M60: peptidase/mucinase domain. b, Purified rSslE forms a clear ring of aggregated protein when incubated in citrate-phosphate buffer between $\mathrm{pH} 4.0$ and 4.4 (upper panel; white asterisks). The ring formed above the liquid line is due to shaking of the sample and it resists solubilization up to $\mathrm{pH} 6.2$ once formed (lower panel; purple asterisk), although some loss of protein band is observed after pH 5.8 (lower panel; red asterisks). c, Increase in ThT fluorescence emission on binding to rSslE fibres formed in citrate-phosphate buffer over $\mathrm{pH} 4.0$ to 8.0. d, Immunoblot of rSslE incubated in citrate-phosphate buffer from $\mathrm{pH} 3.8$ to 4.8 and detected with anti-His 6 antibody. rSslE present in the well represents aggregated protein. e, Far-UV CD spectra of soluble rSslE at pH 4.4 (solid 
line) and 7.4 (dashed line). Secondary structure analysis is shown in the doughnut chart. f, ATR FTMIR spectrum of the amide I region of rSslE fibres, displaying relatively high and low intensity absorption bands at $\sim 1624$ and $1695 \mathrm{~cm}^{-1}$ respectively, characteristic of an anti-parallel $\beta$-sheet structure. 


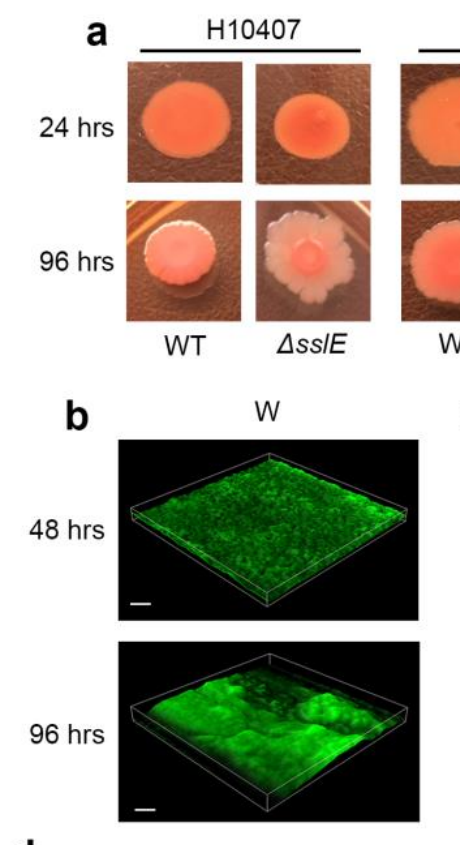

d

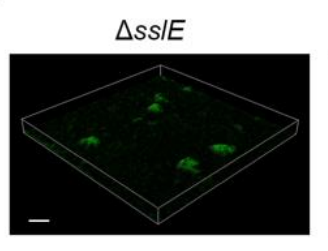

W

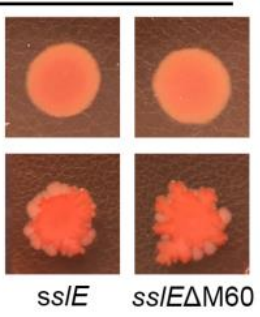

$\Delta s s I E$
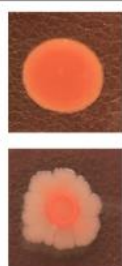

WT
C

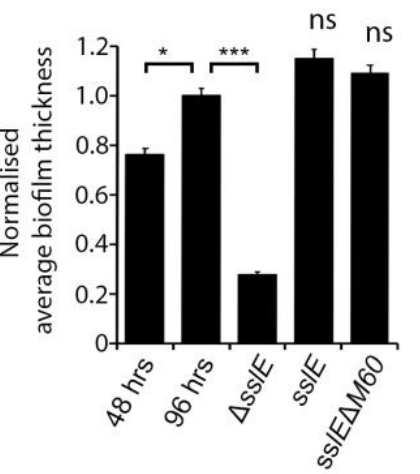

e

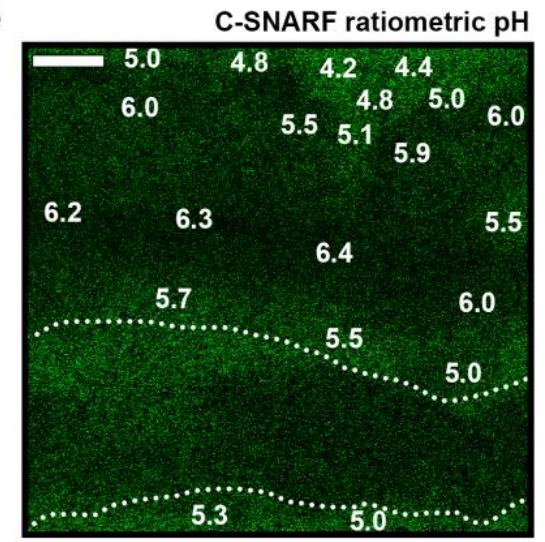

C-SNARF bright field

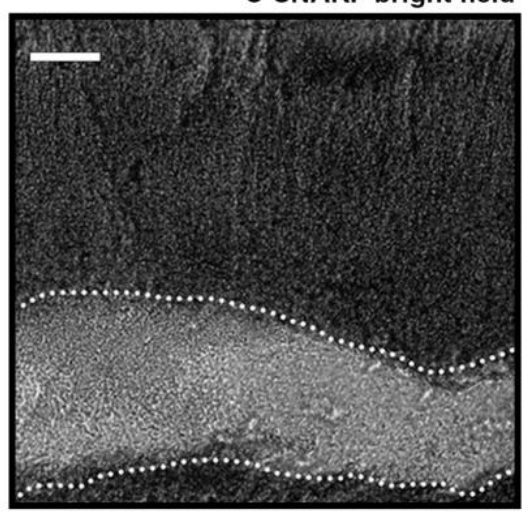

Fig. 2 | Analysis of SslE-dependent biofilm formation by E. coli W and H10407 strains. a, Macrocolony biofilm phenotype of wild-type E. coli W and H10407 strains and their derivatives on Congo red agar medium after 24 and 96 hrs of growth. The derivatives $s s l E$ and sslEAM60 represent trans-complementation of intact $s s l E$ or $s s l E$ minus its M60 domain, respectively, into the $E$. coli W $\Delta s s l E$ mutant. b, CLSM images of wild-type E. coli W biofilms grown for 48 and $96 \mathrm{hrs}$ and stained with lipophilic probe FM 1-43 (green). c, Quantification of 3-dimensional biofilm intensity of wildtype $E$. coli $\mathrm{W}$ biofilms and its derivatives carried out in Image $\mathrm{J}^{40} .{ }^{*} P<0.05 ;{ }^{* * *} P<0.001$; verses W 96 hrs growth by two-tailed Student's t-test. d, CLSM images of E. coli W $\Delta s s l E$ mutant and transcomplementation with $s s l E(\Delta s s l E:: s s l E)$ and $s s l E \Delta \mathrm{M} 60$ (AsslE::sslEAM60) stained with FM 1-43 (green). e, The $\mathrm{pH}$ across wild-type E. coli $\mathrm{W}$ biofilms grown for $96 \mathrm{hrs}$ was monitored ratiometrically using C-SNARF-4 (green). $\mathrm{pH}$ values were calculated over $\sim 30 \mu \mathrm{m}^{2}$ boxes and $\mathrm{pH}$ values for representative regions within the biofilm fringes and centres are annotated. Dotted line outlines water channel within the biofilm, where $\mathrm{pH}$ values are not shown. Scale bar represents $20 \mu \mathrm{m}$. All data are representative of at least three independent experiments. 

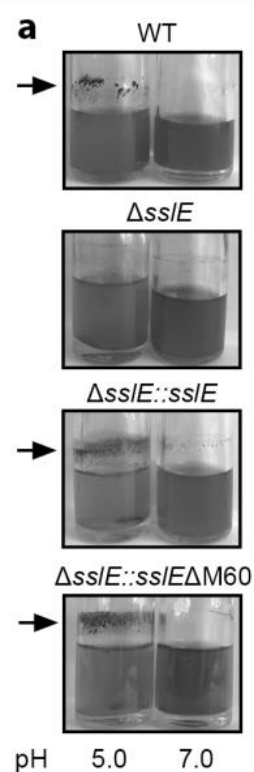

b

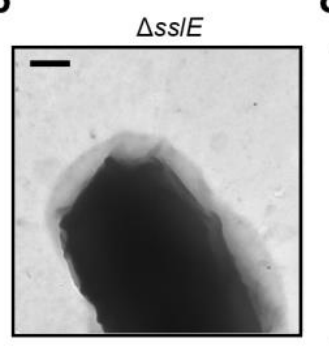

e

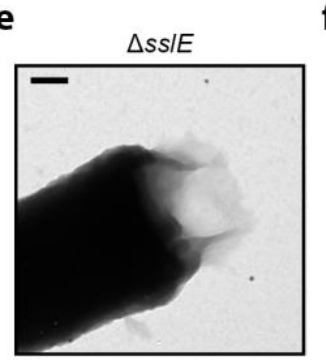

d
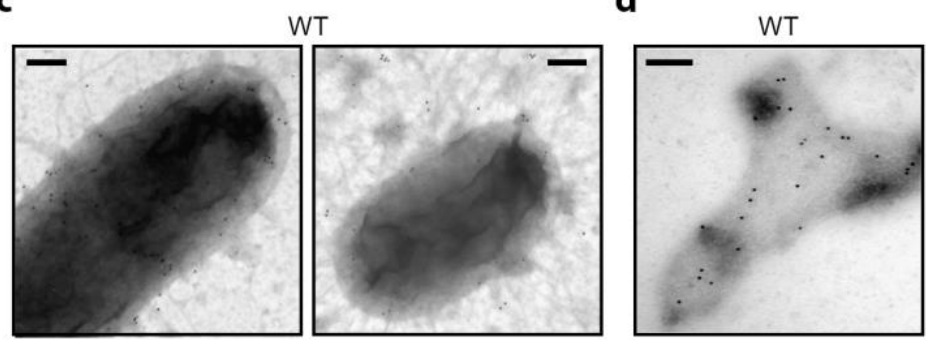

f

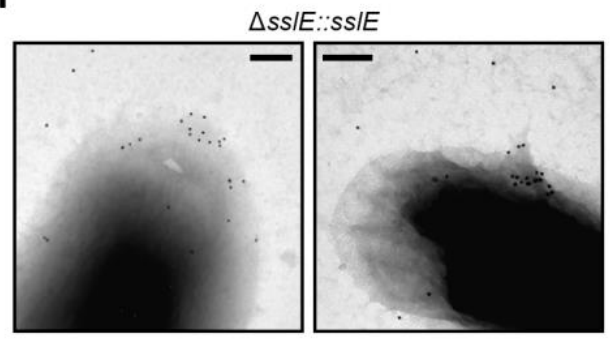

Fig. 3 | Analysis of SslE aggregation after bacterial secretion. a, Accumulation of SslE aggregates on the side of glass tubes containing overnight cultures of E. coli $\mathrm{W}$ strains, grown in LB media at $\mathrm{pH}$ 5.0 or 7.0. Arrows indicate Congo red stained ring containing SslE. b-f, SslE localization upon secretion from $E$. coli $\mathrm{W}$ strains assessed by immunoelectron microscopy. Bacteria were reacted with primary $\alpha-$ rSslE antibodies and secondary gold-labelled antibodies on carbon coated nickel grids and then negatively stained with uranyl acetate. b, e, E. coli W $\Delta s s l E$ mutant washed in citrate-phosphate buffer at $\mathrm{pH} 5.0$ or 4.0, respectively. c, Wild-type $\mathrm{W}$ strain washed in citrate-phosphate buffer at $\mathrm{pH} 5.0$ with $\alpha$-rSslE antibodies reacting with the bacterial surface and secreted fibrous material. d, Image highlighting clustering of antibodies to an extracellular aggregate. f, $\Delta s s l E:: s s l E \mathrm{~W}$ strain washed in citrate-phosphate buffer at $\mathrm{pH} 4.0$, again with $\alpha$-rSslE antibodies reacting with the bacterial surface and secreted fibrous material. Scale bar is equivalent to $200 \mathrm{~nm}$. 

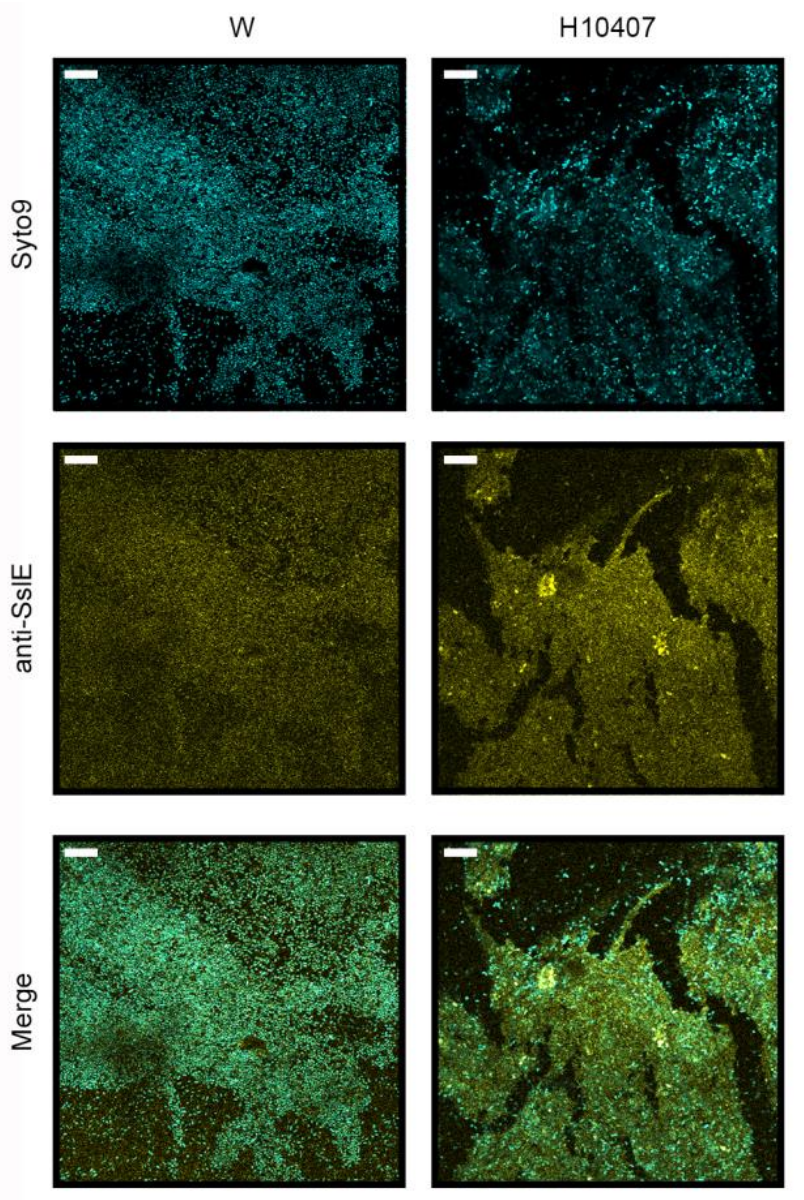

Fig. 4 | Localization of SslE within E. coli biofilms. Wild-type E. coli W and H10407 biofilms were formed using a continuous flow cell system over $96 \mathrm{hrs.} \mathrm{Immunofluorescence} \mathrm{were} \mathrm{performed} \mathrm{on} \mathrm{fixed}$ biofilms wherein the biofilms were incubated with $\alpha$-rSslE antibodies (1:200 dilution) then incubate with goat anti-rabbit conjugated Alexa Fluor 633 (yellow). DNA was stained with Syto9 (cyan) to identify bacteria and eDNA within the biofilm matrix. Biofilms were visualized by CLSM. Scale bar is equivalent to $10 \mu \mathrm{m}$. All data are representative of three independent experiments. 

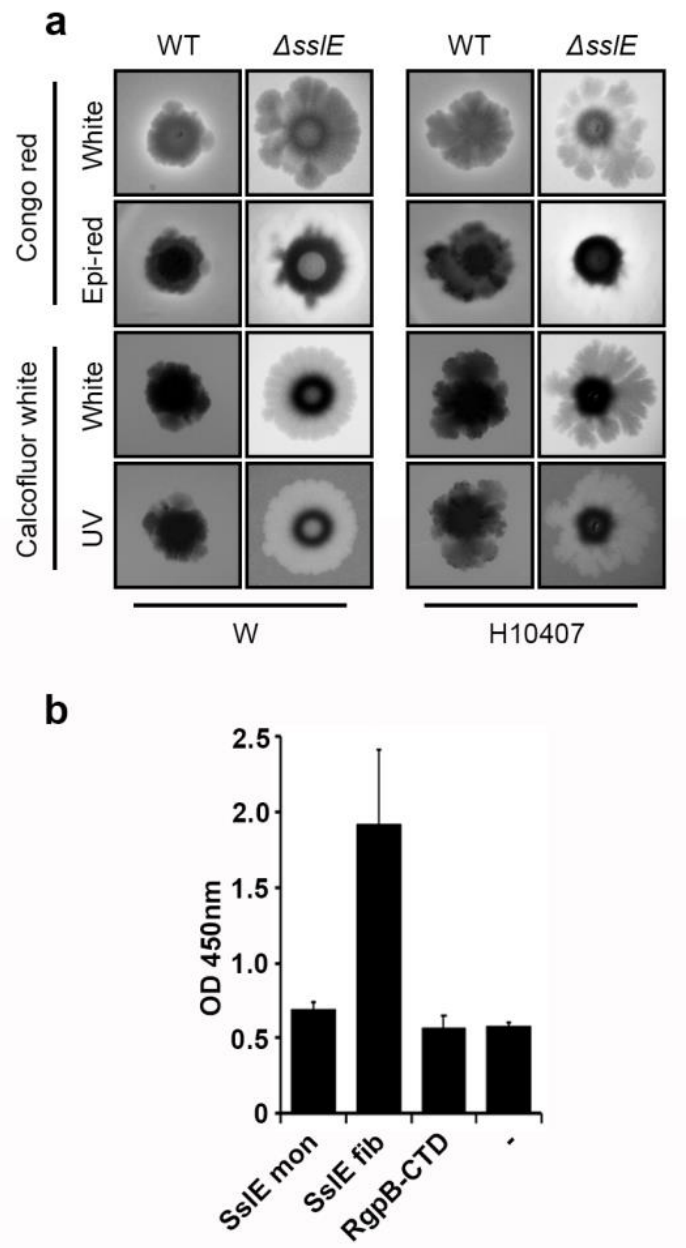

Fig. 5 | Binding properties of SslE aggregates. a, Macrocolony biofilm phenotype of wild-type and $\Delta s s l E$ mutant $E$. coli $\mathrm{W}$ and $\mathrm{H} 10407$ strains on Congo red or calcofluor white agar medium after $96 \mathrm{hrs}$ of growth. Macrocolonies were visualized with white light to determine the edge of the colony, and then either epi-red or UV light was used to determine the localization of Congo red or calcofluor white, respectively, within the colony. $\mathbf{b}$, ELISA analysis of binding between cellulose discs and His-tagged rSslE monomers, His-tagged rSslE fibres and His-tagged P. gingivalis RgpB-CTD control, at pH 6.0 and detected with anti-His 6 antibody. BSA-coated wells were used as controls. ${ }^{*} P<0.05$; verses control empty well (-) by two-tailed Student's test. All data are representative of at least three independent experiments performed in triplicate. 

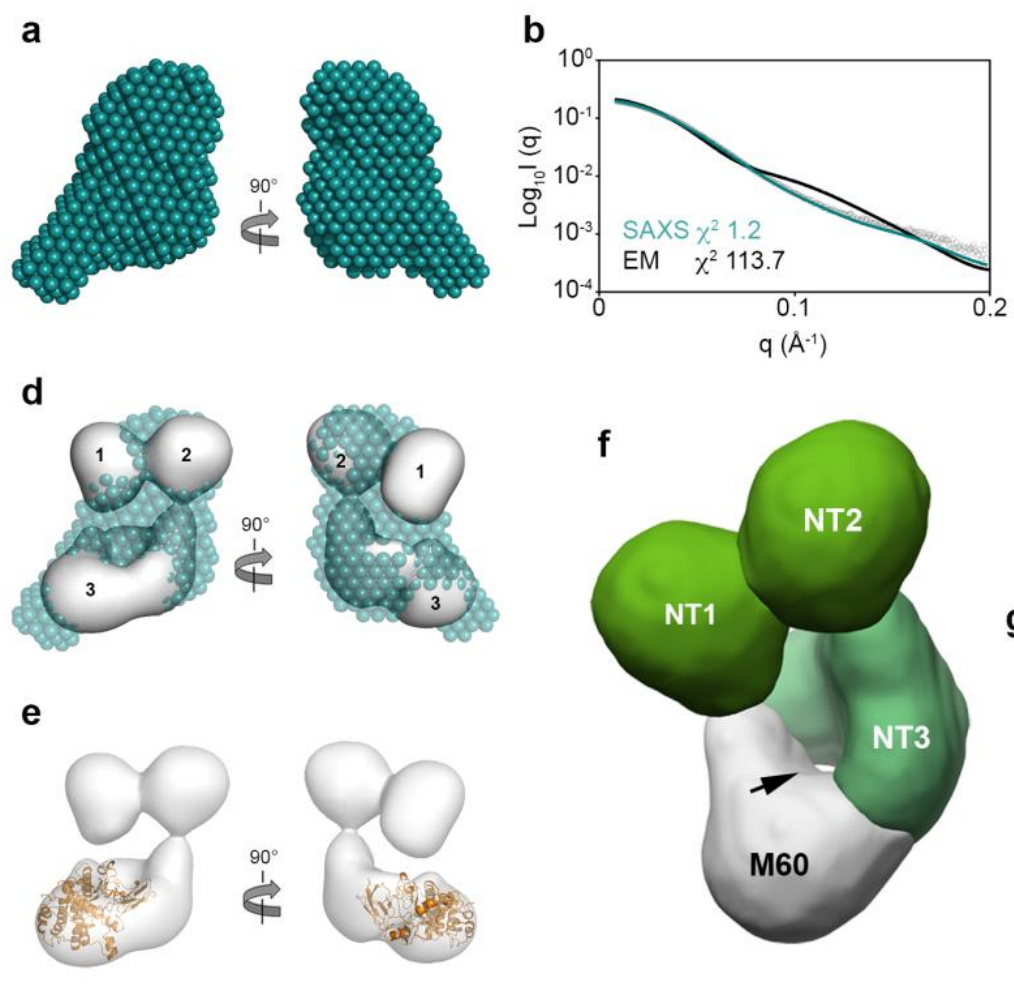
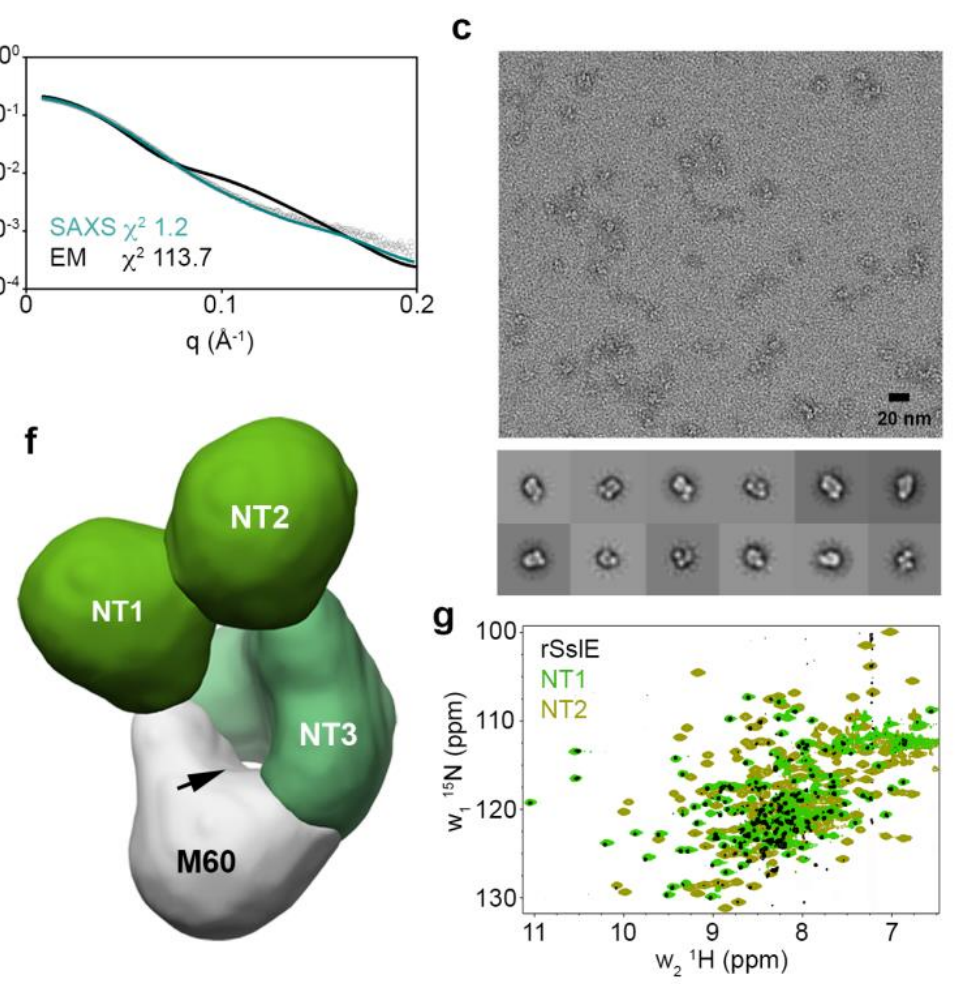

Fig. 6 Structural features of monomeric SsIE. a, SAXS bead model of rSslE at pH 7.4. b, Fit of the SAXS bead model (teal line) and negative-stain TEM map (black line) of rSslE to the rSslE SAXS data (black open circles) with $\chi^{2}$ of 1.2 and 113.7, respectively. c, Representative negative-stain TEM micrograph of rSslE (pH 7.4) at 50,000× nominal magnification with representative 2D classifications. Scale bar represents $20 \mathrm{~nm}$. d, Overlay of the 22 A resolution TEM map (grey) and SAXS bead model (teal) with the three defined regions in SslE highlighted. e, Docking of an SslE M60 domain homology model (orange) into the TEM map. f, TEM map of rSslE colored based on domain organization. The NT3-M60 interdomain channel is highlighted with an arrow. g, TROSY ${ }^{1} \mathrm{H}^{15} \mathrm{~N}-\mathrm{HSQC}$ spectrum of rSslE (black) overlaid with ${ }^{1} \mathrm{H}^{15} \mathrm{~N}-\mathrm{HSQC}$ spectra of the SslE NT1 (light green) and NT2 (olive green) domains. 
a

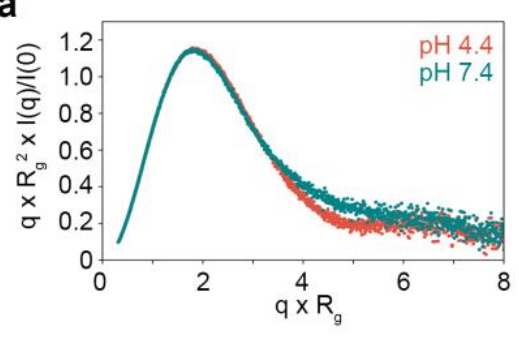

b
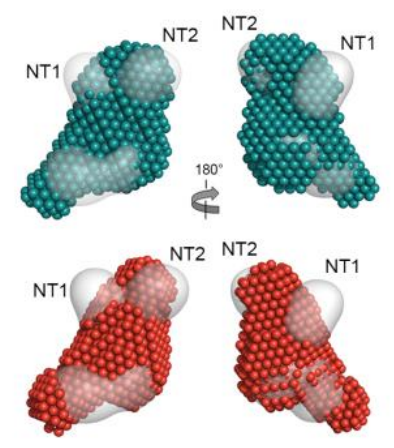

C

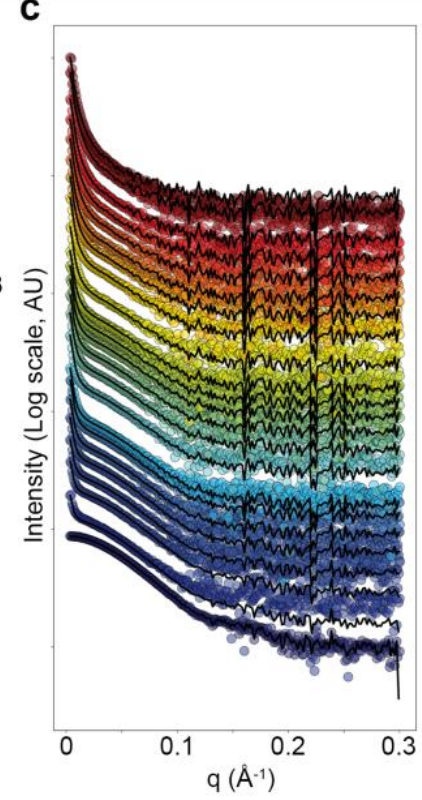

d
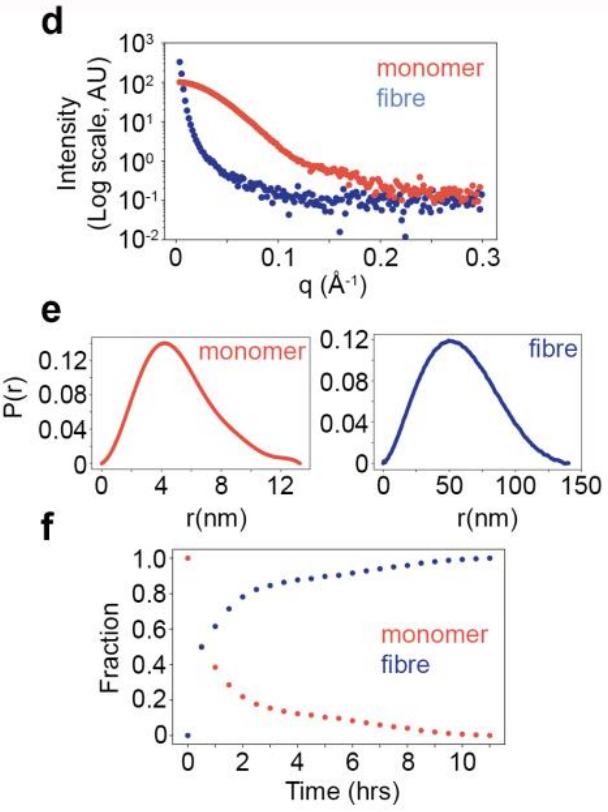

Fig. 7 | Kinetics of SslE aggregation. a, SAXS normalized Kratky plots for rSslE at pH 4.4 and 7.4. b, Overlay of rSslE SAXS bead models at pH 4.4 (red) and 7.4 (teal) with rSslE TEM map (grey). NT1 and NT2 domains are highlighted. c, COSMiCS decomposition of rSslE SAXS fibrillation data, assuming the co-existence of two components. SEC-SAXS curve for rSslE at $\mathrm{pH} 4.4$ is also shown for $\mathrm{t}=0$. d, COSMiCS decomposed scattering curves for rSslE monomer (red) and aggregates (blue). e, Shape distribution $[P(r)]$ functions derived from COSMiCS decomposed scattering curves for rSslE monomer (red) and aggregates (blue). f, Relative populations of the two species of rSslE derived from the COSMiCS analysis over time. 

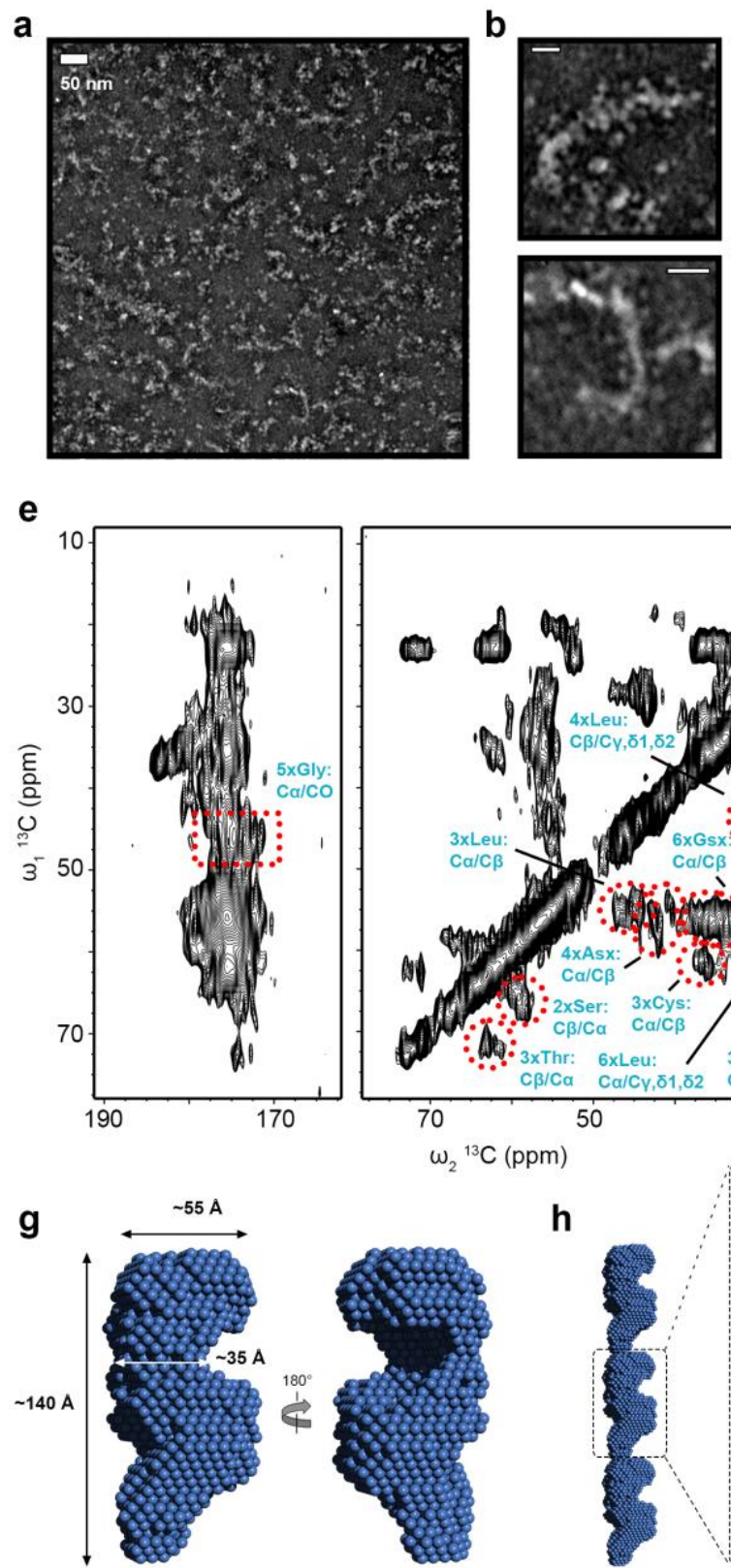

$\mathbf{h}$

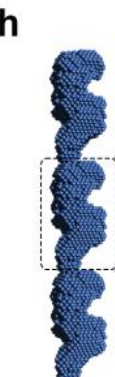

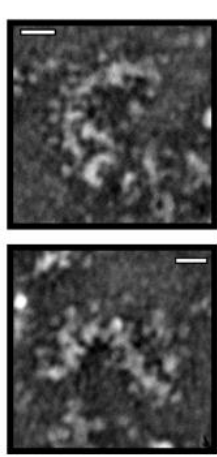
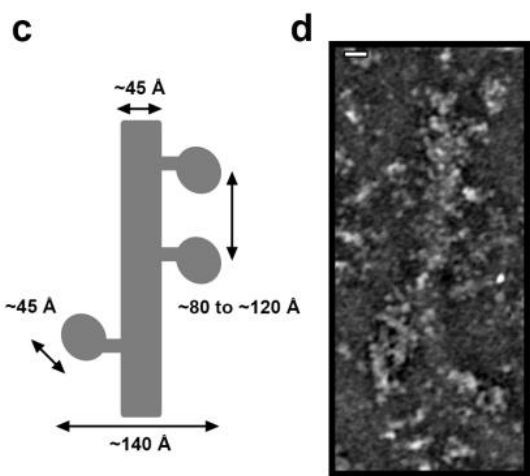

f
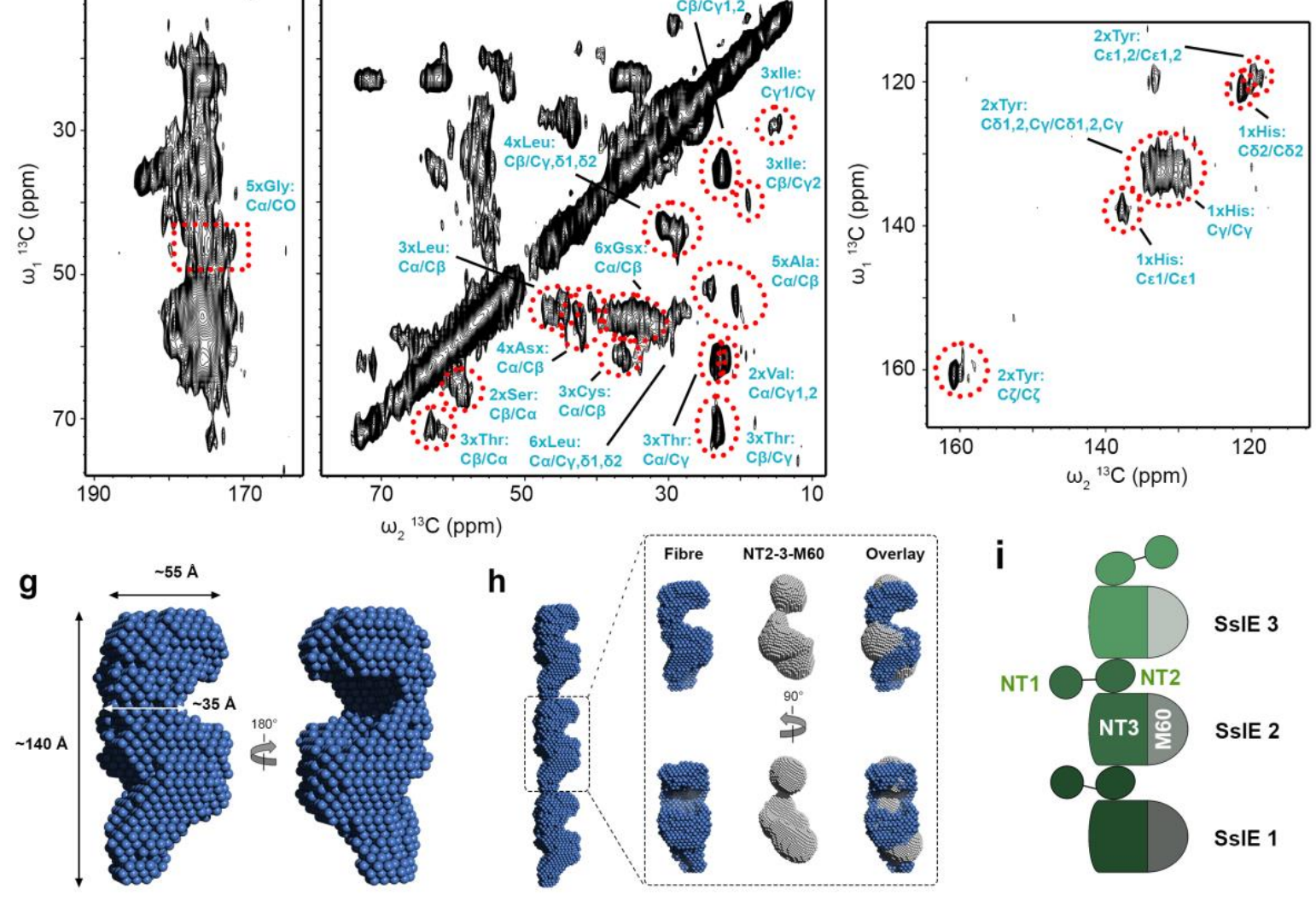

Fig. 8 | Model of SslE fibres. a, TEM analysis of negatively stained SslE fibres. Two major species are observed. b, Smaller filaments have an average width of $\sim 4.5 \mathrm{~nm}$ and are coated with globular structures of $\sim 4.5 \mathrm{~nm}$ in diameter. Scale bar, $20 \mathrm{~nm}$. c, Schematic describing the overall dimensions of the smaller SslE fibre species. d, Larger species of SslE fibres appear as 20 to $40 \mathrm{~nm}$ wide structures with variable lengths. Scale bar, $20 \mathrm{~nm}$. e, f, DARR ssNMR spectrum of SslE fibres focussed on the carbonyl (e, left), aliphatic (e, right) and aromatic (f) regions. Likely amino acid type assignments are highlighted with red dashed lines and annotated in teal. g, SAXS bead model for the repeating unit of the SslE fibre core with overall dimensions given. $\mathbf{h}$, Three units of the SslE fibre core translated along 
the fibre axis (left). One unit is zoomed in and overlaid with a bead model created of the rSslE negativestain TEM map with the NT1 domain removed (NT2-3-M60; right). i, Proposed model for SslE fibres. The core of the fibre contains the NT2, NT3 and M60 domains and is decorated with flexible NT1. The core fibre is formed via interactions between the NT2 and NT3 domains of adjacent SslE molecules. 